\title{
Evaluation Metrics
}

\author{
Jaime Arguello \\ INLS 509: Information Retrieval \\ jarguell@email.unc.edu
}

March 25, 2013 


\section{Batch Evaluation evaluation metrics}

- At this point, we have a set of queries, with identified relevant and non-relevant documents

- The goal of an evaluation metric is to measure the quality of a particular ranking of known relevant/non-relevant documents 


\section{Set Retrieval precision and recall}

- So far, we've defined precision and recall assuming boolean retrieval: a set of relevant documents (REL) and a set of retrieved documents (RET)

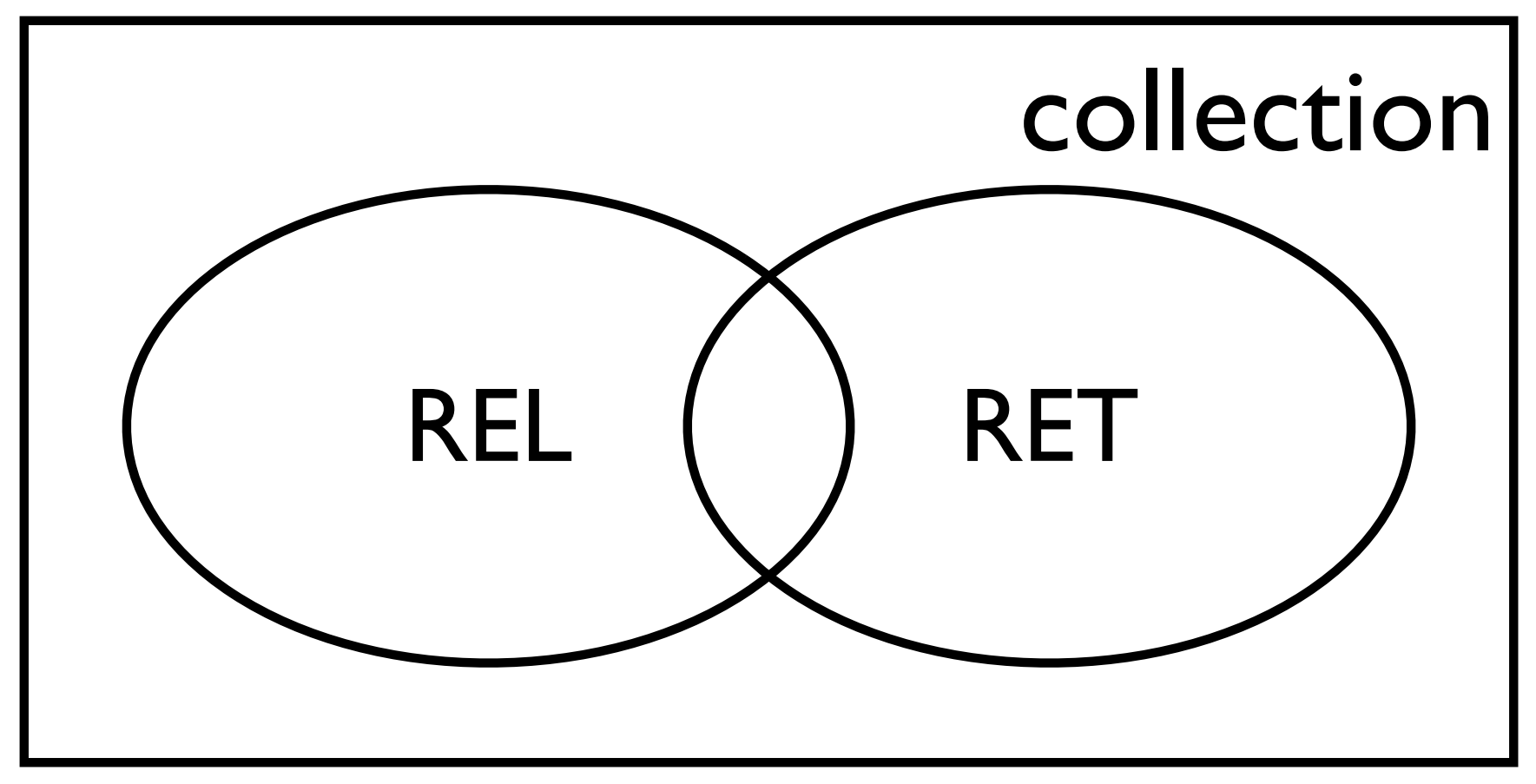




\section{Set Retrieval precision and recall}

- Precision (P): the proportion of retrieved documents that are relevant

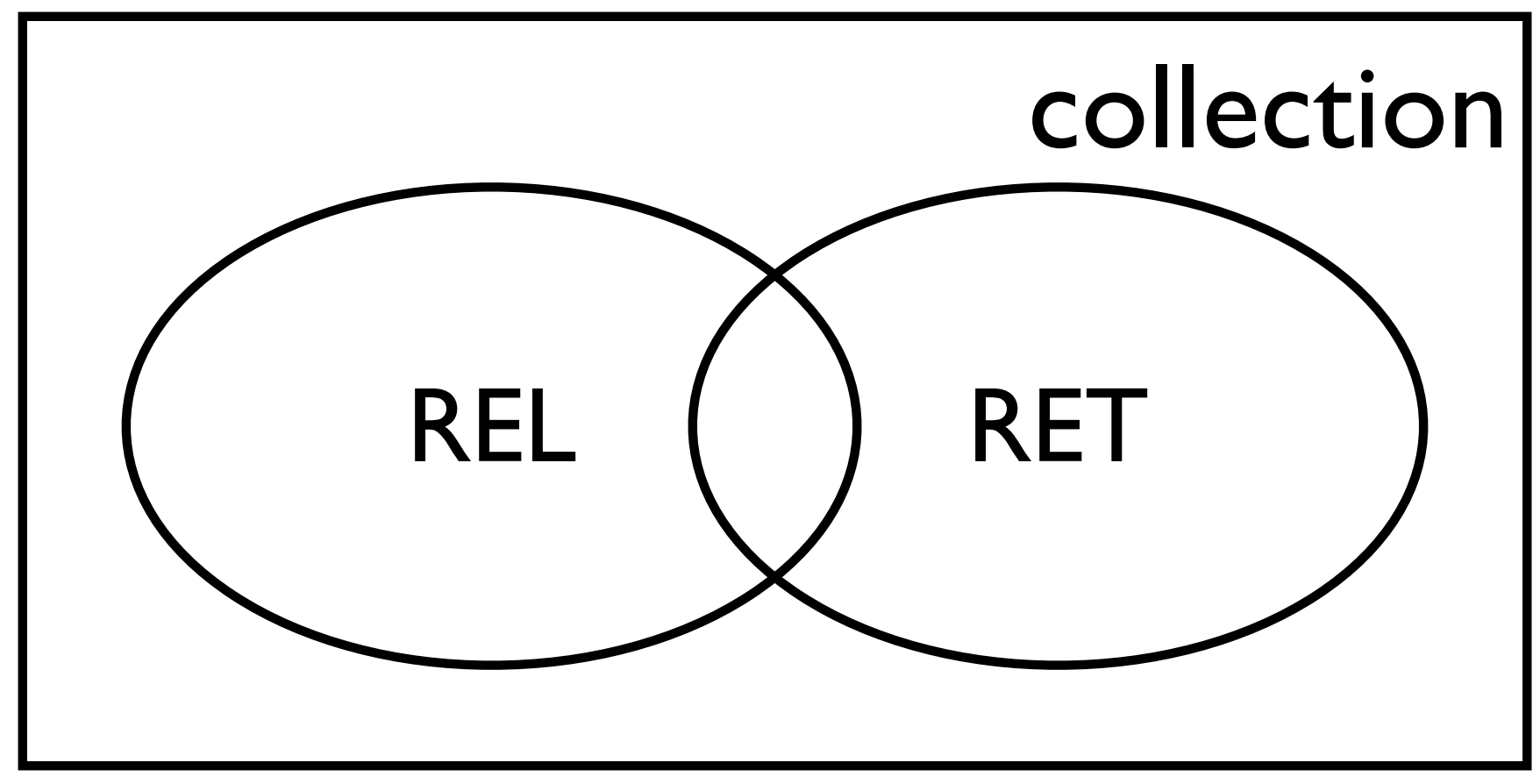

$$
\mathcal{P}=\frac{|R E T \cap R E L|}{|R E T|}
$$




\section{Set Retrieval precision and recall}

- Recall (R): the proportion of relevant documents that are retrieved

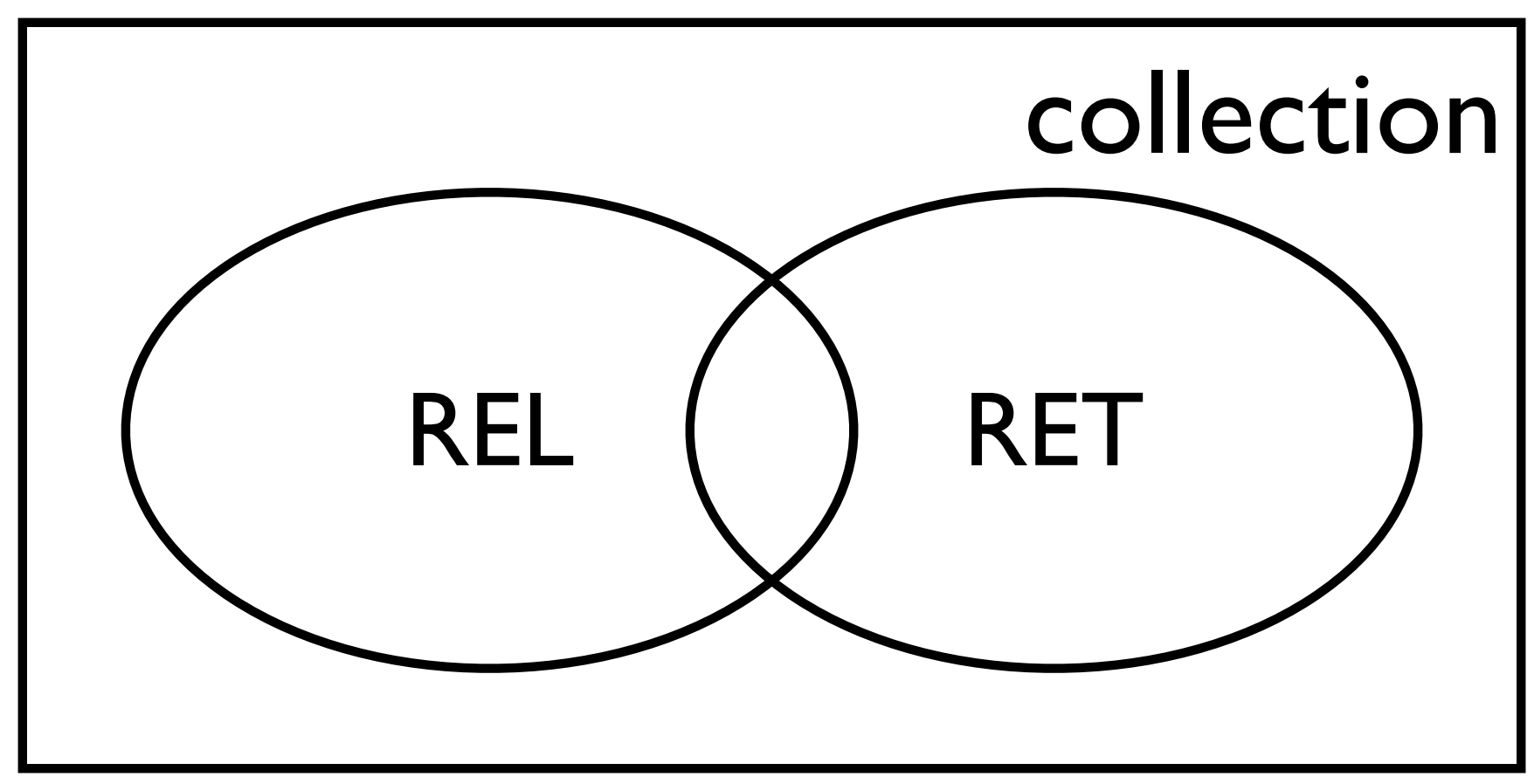

$$
\mathcal{R}=\frac{|R E T \cap R E L|}{|R E L|}
$$




\section{Set Retrieval precision and recall}

- Recall measures the system's ability to find all the relevant documents

- Precision measures the system's ability to reject any nonrelevant documents in the retrieved set 


\section{Set Retrieval precision and recall}

- A system can make two types of errors:

- a false positive error: the system retrieves a document that is non-relevant (should not have been retrieved)

- a false negative error: the system fails to retrieve a document that is relevant (should have been retrieved)

- How do these types of errors affect precision and recall? 


\section{Set Retrieval precision and recall}

- A system can make two types of errors:

- a false positive error: the system retrieves a document that is non-relevant (should not have been retrieved)

- a false negative error: the system fails to retrieve a document that is relevant (should have been retrieved)

- How do these types of errors affect precision and recall?

- Precision is affected by the number of false positive errors

- Recall is affected by the number of false negative errors 


\section{Set Retrieval combining precision and recall}

- Oftentimes, we want a system that has high-precision and high-recall

- We want a metric that measures the balance between precision and recall

- One possibility would be to use the arithmetic mean:

$$
\operatorname{arithmetic} \operatorname{mean}(\mathcal{P}, \mathcal{R})=\frac{\mathcal{P}+\mathcal{R}}{2}
$$

- What is problematic with this way of summarizing precision and recall? 


\section{Set Retrieval combining precision and recall}

- It's easy for a system to "game" the arithmetic mean of precision and recall

- Bad: a system that obtains I.0 precision and near 0.0 recall would get a mean value of about 0.50

- Bad: a system that obtains 1.0 recall and near 0.0 precision would get a mean value of about 0.50

- Better: a system that obtains 0.50 precision and near 0.50 recall would get a mean value of about 0.50 


\section{Set Retrieval \\ F-measure (also known as F1)}

- A system that retrieves a single relevant document would get $\mathrm{I} .0$ precision and near 0.0 recall

- A system that retrieves the entire collection would get I.0 recall and near 0.0 precision

- Solution: use the harmonic mean rather than the arithmetic mean

- F-measure:

$$
\mathcal{F}=\frac{1}{\frac{1}{2}\left(\frac{1}{\mathcal{P}}+\frac{1}{\mathcal{R}}\right)}=\frac{2 \times \mathcal{P} \times \mathcal{R}}{\mathcal{P}+\mathcal{R}}
$$




\section{Set Retrieval \\ F-measure (also known as F1)}

- The harmonic mean punishes small values
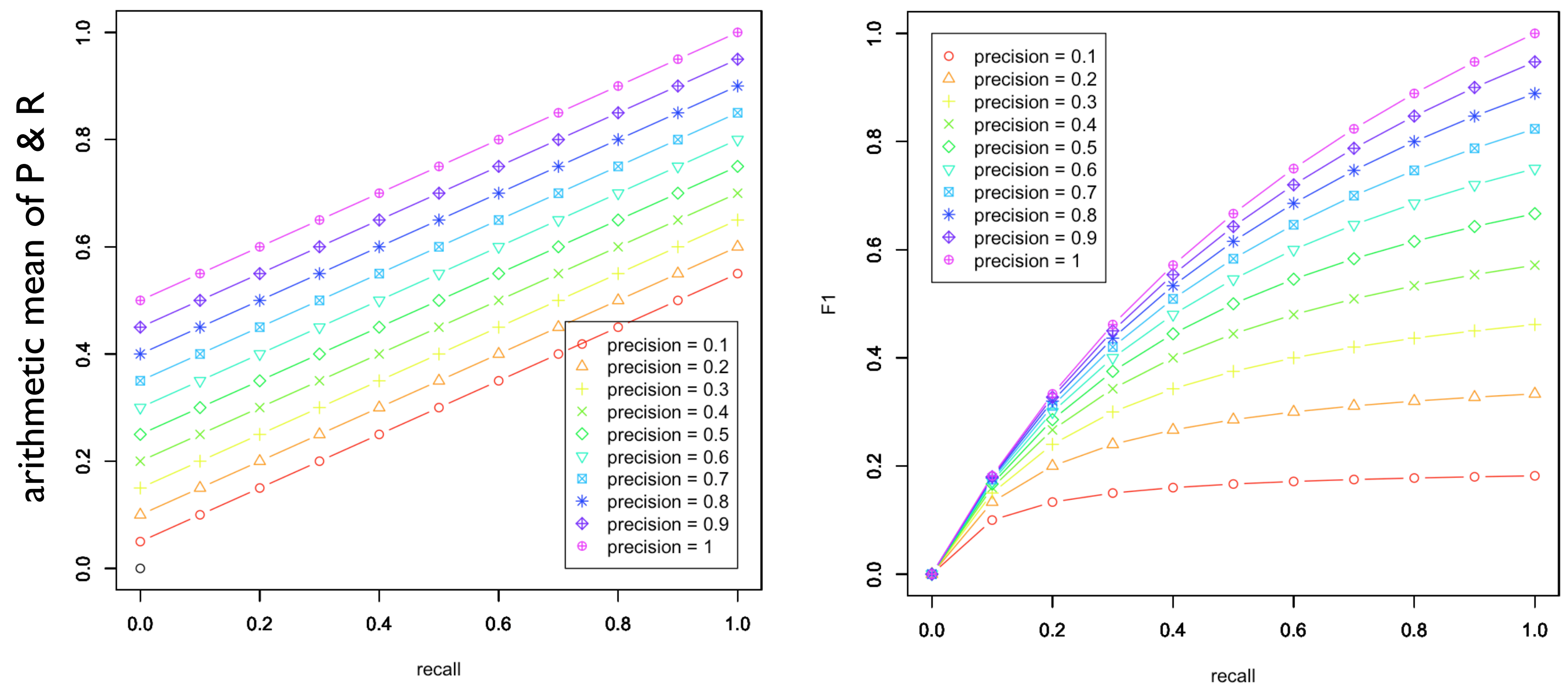

(slide courtesy of Ben Carterette) 


\section{Ranked Retrieval precision and recall}

- In most situations, the system outputs a ranked list of documents rather than an unordered set

- User-behavior assumption:

- The user examines the output ranking from top-tobottom until he/she is satisfied or gives up

- Precision and recall can also be used to evaluate a ranking

- Precision/Recall @ rank K 


\section{Ranked Retrieval precision and recall}

- Precision: proportion of retrieved documents that are relevant

- Recall: proportion of relevant documents that are retrieved

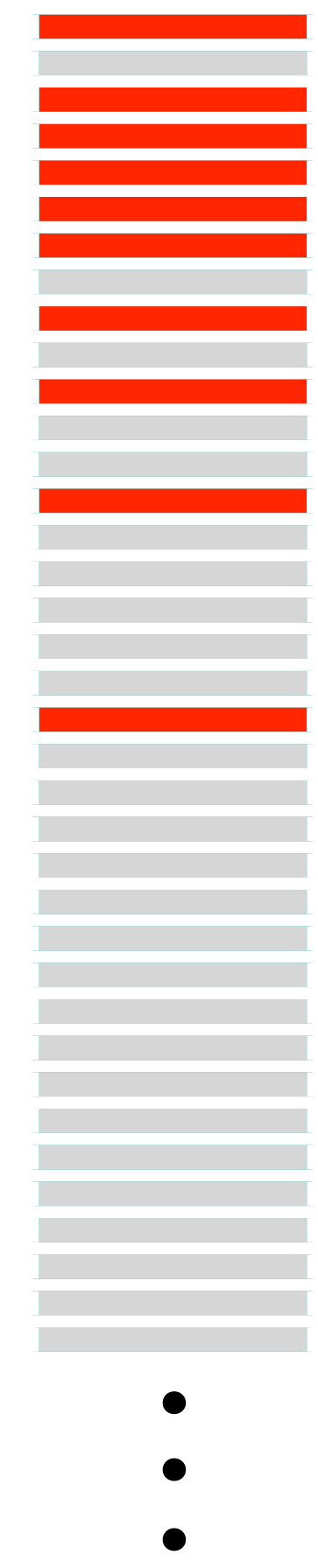




\section{Ranked Retrieval}

precision and recall

- P@K: proportion of retrieved top-K documents that are relevant

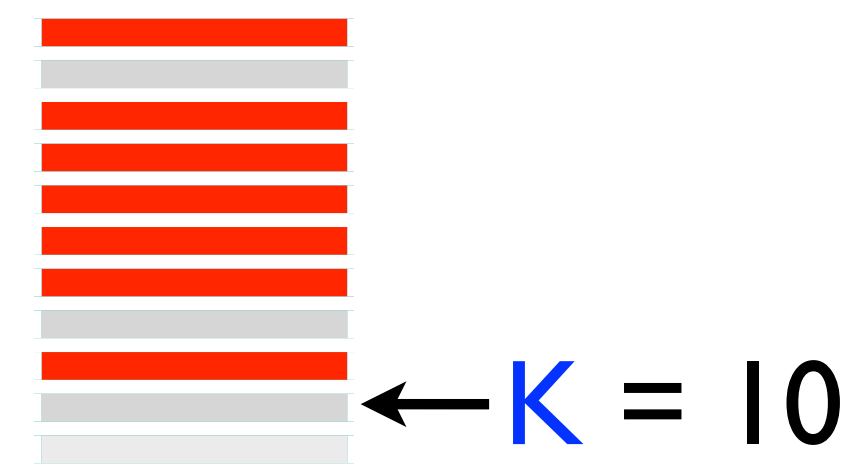

- R@K: proportion of relevant documents that are retrieved in the top-K

- Assumption: the user will only examine the top- $\mathrm{K}$ results

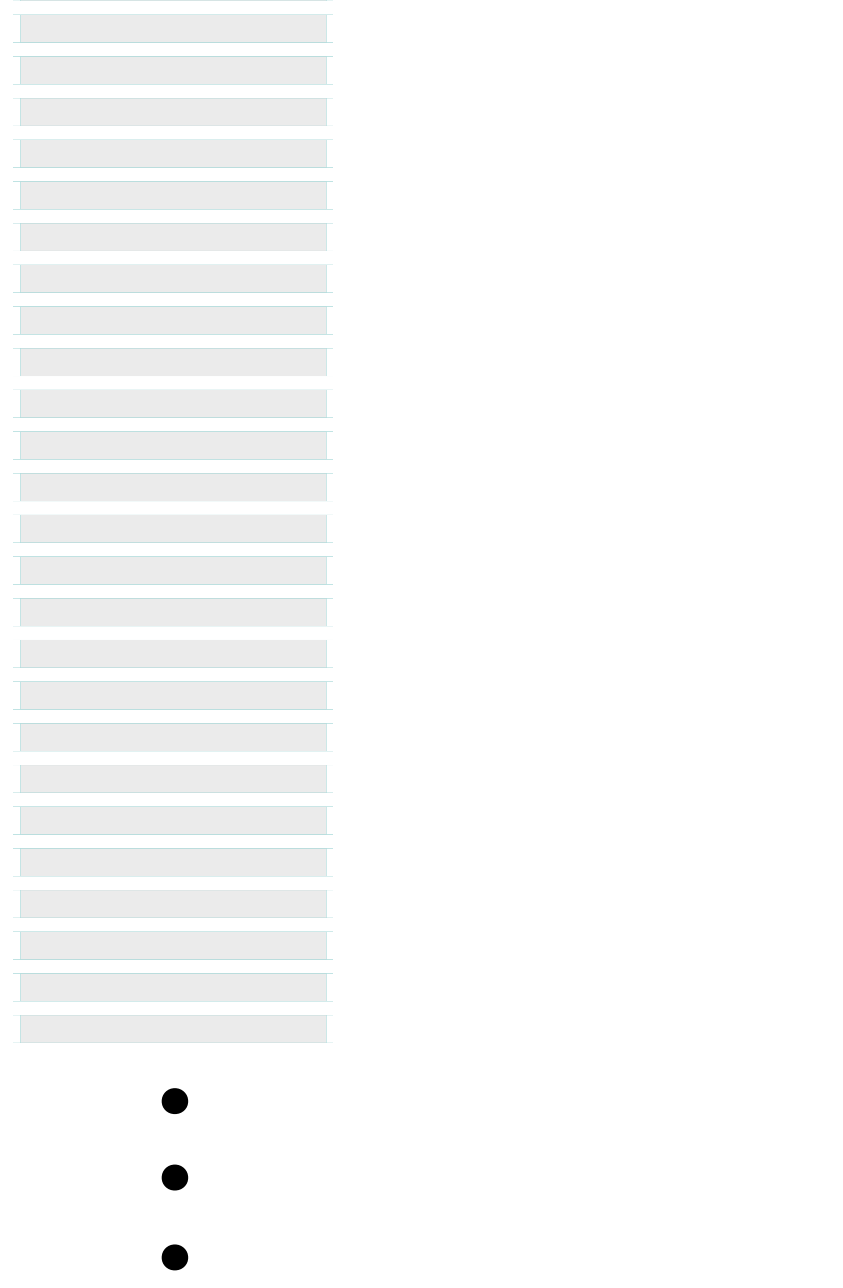




\section{Ranked Retrieval}

precision and recall: exercise

- Assume 20 relevant documents

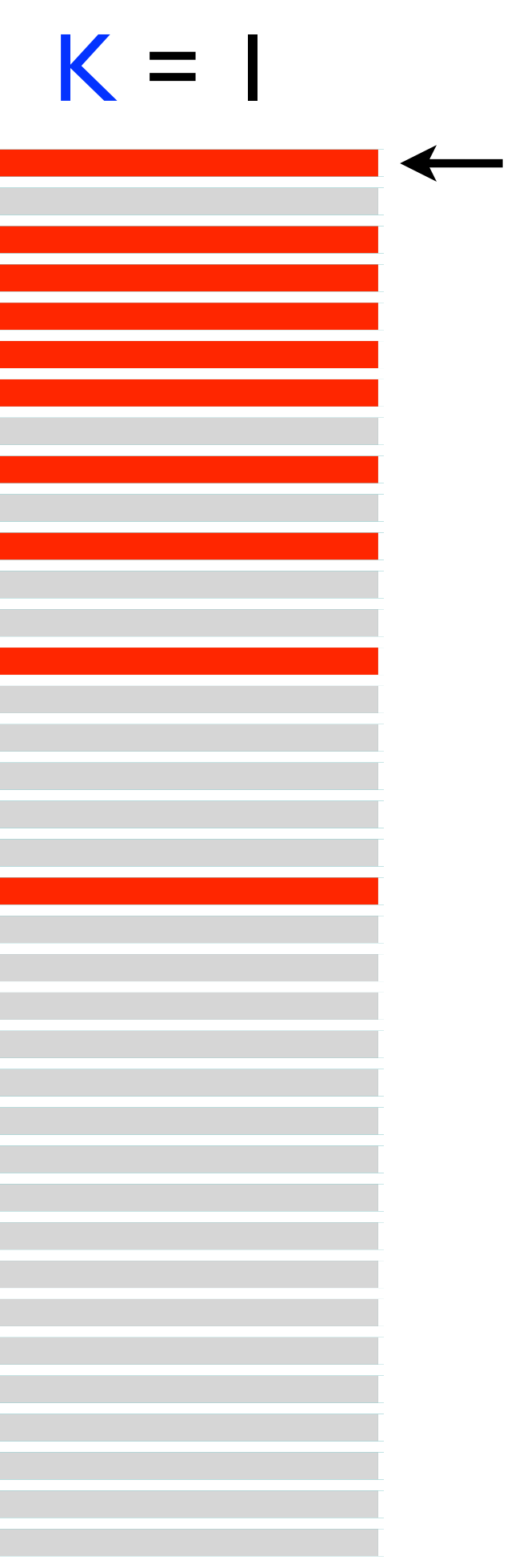




\section{Ranked Retrieval}

precision and recall: exercise

- Assume 20 relevant documents

$K=2$

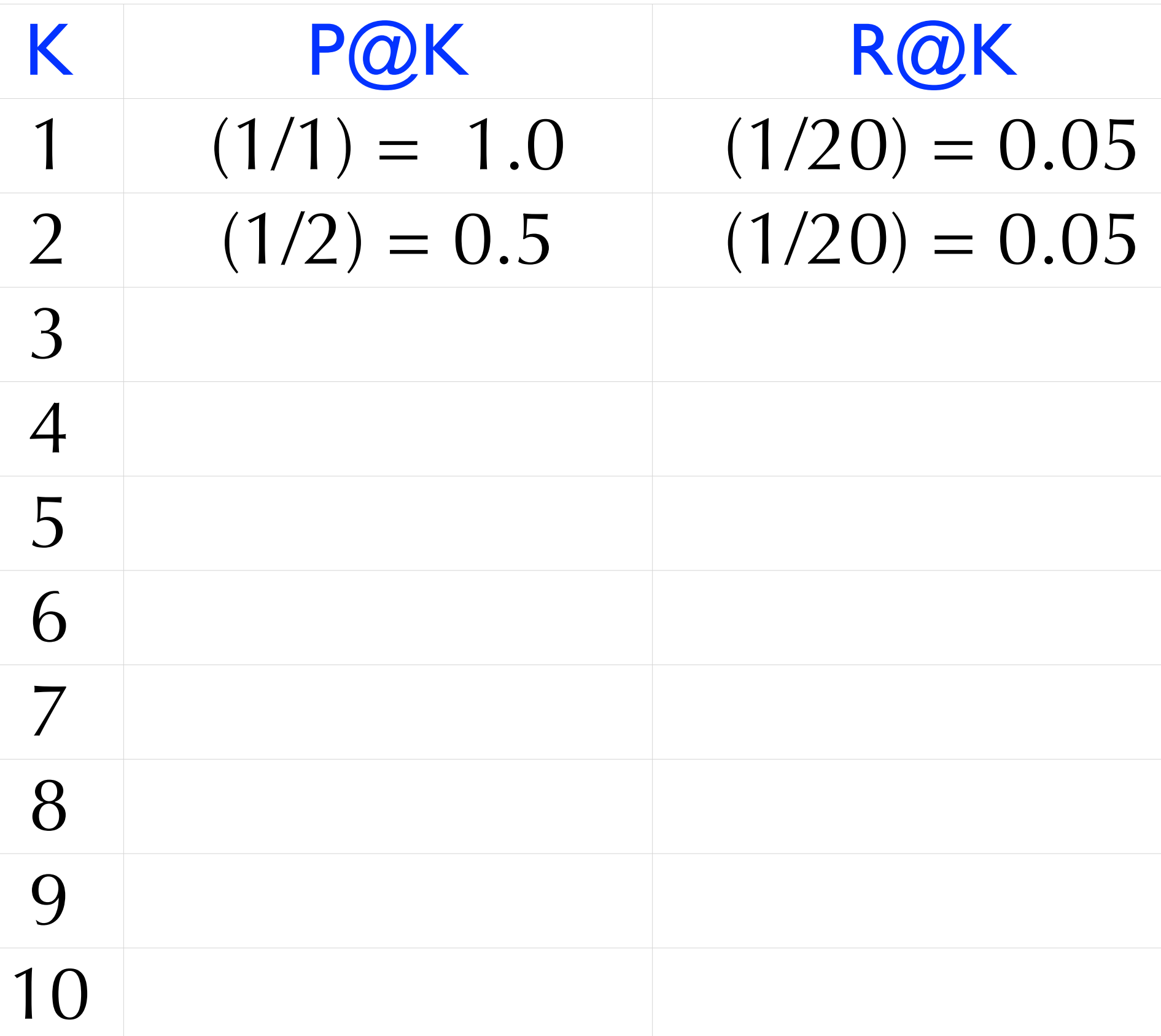




\section{Ranked Retrieval} precision and recall: exercise

- Assume 20 relevant documents

$$
K=3
$$

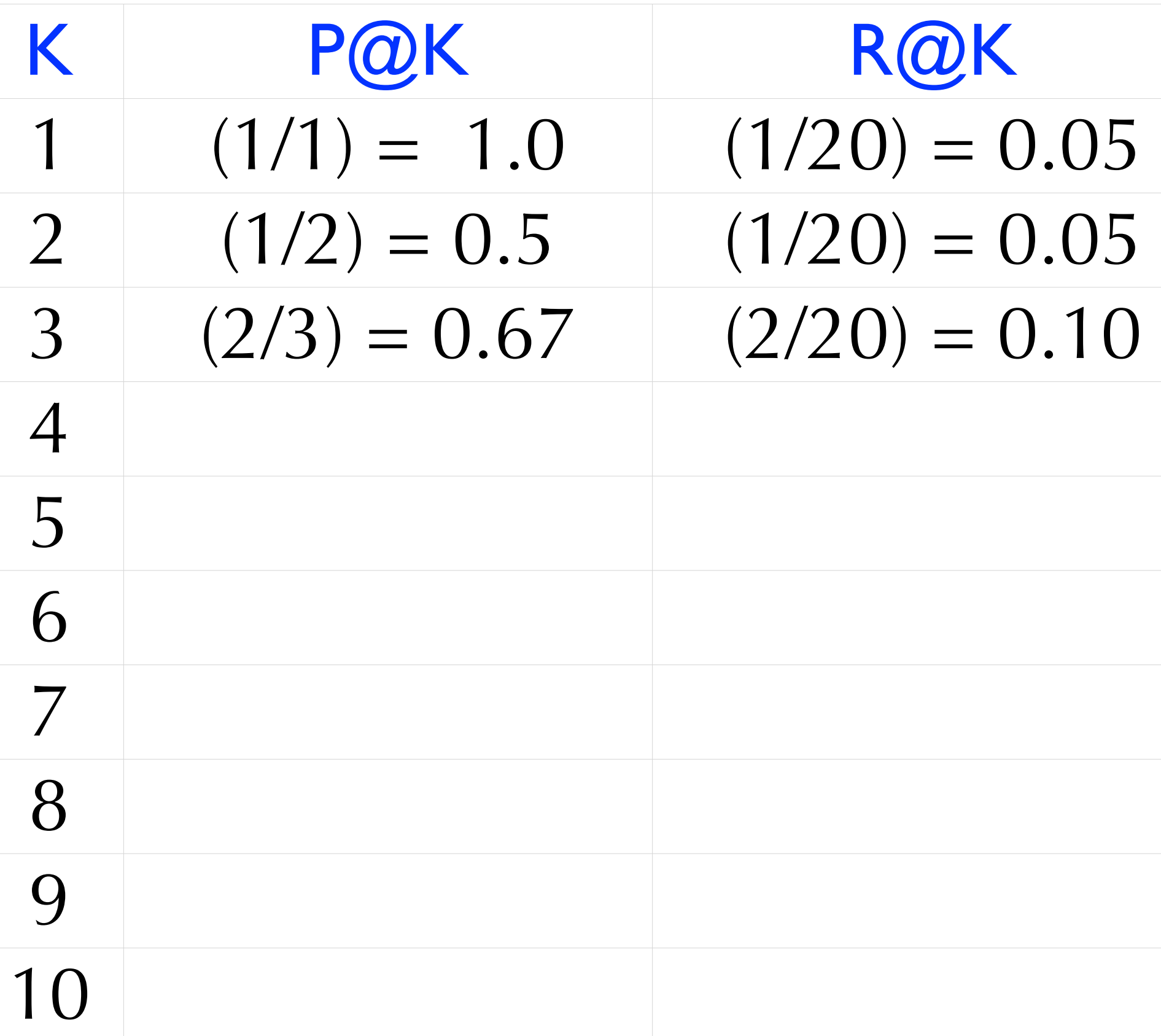




\section{Ranked Retrieval} precision and recall: exercise

- Assume 20 relevant documents

$$
K=4
$$

$\begin{array}{ccc}\text { K } & \text { P@K } & \text { R@K } \\ 1 & (1 / 1)=1.0 & (1 / 20)=0.05 \\ 2 & (1 / 2)=0.5 & (1 / 20)=0.05 \\ 3 & (2 / 3)=0.67 & (2 / 20)=0.10 \\ 4 & (3 / 4)=0.75 & (3 / 20)=0.15 \\ 5 & & \\ 6 & & \\ 7 & & \\ 8 & & \\ 9 & & \\ 10 & & \end{array}$




\section{Ranked Retrieval} precision and recall: exercise

- Assume 20 relevant documents

$$
K=5
$$

$\begin{array}{ccc}\text { K } & \text { P@K } & \text { R@K } \\ 1 & (1 / 1)=1.0 & (1 / 20)=0.05 \\ 2 & (1 / 2)=0.5 & (1 / 20)=0.05 \\ 3 & (2 / 3)=0.67 & (2 / 20)=0.10 \\ 4 & (3 / 4)=0.75 & (3 / 20)=0.15 \\ 5 & (4 / 5)=0.80 & (4 / 20)=0.20 \\ 6 & & \\ 7 & & \\ 8 & & \\ 9 & & \\ 10 & & \end{array}$




\section{Ranked Retrieval}

\section{precision and recall: exercise}

- Assume 20 relevant documents

$$
K=6
$$

$\begin{array}{ccc}\mathrm{K} & \mathrm{P} @ \mathrm{~K} & \mathrm{R} @ \mathrm{~K} \\ 1 & (1 / 1)=1.0 & (1 / 20)=0.05 \\ 2 & (1 / 2)=0.5 & (1 / 20)=0.05 \\ 3 & (2 / 3)=0.67 & (2 / 20)=0.10 \\ 4 & (3 / 4)=0.75 & (3 / 20)=0.15 \\ 5 & (4 / 5)=0.80 & (4 / 20)=0.20 \\ 6 & (5 / 6)=0.83 & (5 / 20)=0.25 \\ 7 & & \\ 8 & & \\ 9 & & \\ 10 & & \end{array}$




\section{Ranked Retrieval}

precision and recall: exercise

- Assume 20 relevant documents

$\begin{array}{ccc}\text { K } & \text { P@K } & \text { R@K } \\ 1 & (1 / 1)=1.0 & (1 / 20)=0.05 \\ 2 & (1 / 2)=0.5 & (1 / 20)=0.05 \\ 3 & (2 / 3)=0.67 & (2 / 20)=0.10 \\ 4 & (3 / 4)=0.75 & (3 / 20)=0.15 \\ 5 & (4 / 5)=0.80 & (4 / 20)=0.20 \\ 6 & (5 / 6)=0.83 & (5 / 20)=0.25 \\ 7 & (6 / 7)=0.86 & (6 / 20)=0.30 \\ 8 & & \\ 9 & & \\ 10 & & \end{array}$




\section{Ranked Retrieval}

\section{precision and recall: exercise}

- Assume 20 relevant documents

$$
\mathrm{K}=8
$$

$\begin{array}{ccc}\mathrm{K} & \mathrm{P} @ \mathrm{~K} & \mathrm{R} @ \mathrm{~K} \\ 1 & (1 / 1)=1.0 & (1 / 20)=0.05 \\ 2 & (1 / 2)=0.5 & (1 / 20)=0.05 \\ 3 & (2 / 3)=0.67 & (2 / 20)=0.10 \\ 4 & (3 / 4)=0.75 & (3 / 20)=0.15 \\ 5 & (4 / 5)=0.80 & (4 / 20)=0.20 \\ 6 & (5 / 6)=0.83 & (5 / 20)=0.25 \\ 7 & (6 / 7)=0.86 & (6 / 20)=0.30 \\ 8 & (6 / 8)=0.75 & (6 / 20)=0.30 \\ 9 & & \\ 10 & & \end{array}$




\section{Ranked Retrieval}

\section{precision and recall: exercise}

- Assume 20 relevant documents

$$
K=9
$$

$\begin{array}{ccc}\mathrm{K} & \mathrm{P} @ \mathrm{~K} & \mathrm{R} @ \mathrm{~K} \\ 1 & (1 / 1)=1.0 & (1 / 20)=0.05 \\ 2 & (1 / 2)=0.5 & (1 / 20)=0.05 \\ 3 & (2 / 3)=0.67 & (2 / 20)=0.10 \\ 4 & (3 / 4)=0.75 & (3 / 20)=0.15 \\ 5 & (4 / 5)=0.80 & (4 / 20)=0.20 \\ 6 & (5 / 6)=0.83 & (5 / 20)=0.25 \\ 7 & (6 / 7)=0.86 & (6 / 20)=0.30 \\ 8 & (6 / 8)=0.75 & (6 / 20)=0.30 \\ 9 & (7 / 9)=0.78 & (7 / 20)=0.35 \\ 10 & & \end{array}$




\section{Ranked Retrieval}

\section{precision and recall: exercise}

- Assume 20 relevant documents

$\begin{array}{ccc}\mathrm{K} & \mathrm{P} @ \mathrm{~K} & \mathrm{R} @ \mathrm{~K} \\ 1 & (1 / 1)=1.0 & (1 / 20)=0.05 \\ 2 & (1 / 2)=0.5 & (1 / 20)=0.05 \\ 3 & (2 / 3)=0.67 & (2 / 20)=0.10 \\ 4 & (3 / 4)=0.75 & (3 / 20)=0.15 \\ 5 & (4 / 5)=0.80 & (4 / 20)=0.20 \\ 6 & (5 / 6)=0.83 & (5 / 20)=0.25 \\ 7 & (6 / 7)=0.86 & (6 / 20)=0.30 \\ 8 & (6 / 8)=0.75 & (6 / 20)=0.30 \\ 9 & (7 / 9)=0.78 & (7 / 20)=0.35 \\ 10 & (7 / 10)=0.70 & (7 / 20)=0.35\end{array}$




\section{Ranked Retrieval precision and recall}

- Problem: what value of $\mathrm{K}$ should we use to evaluate?

- Which is better in terms of P@I0 and R@I0?
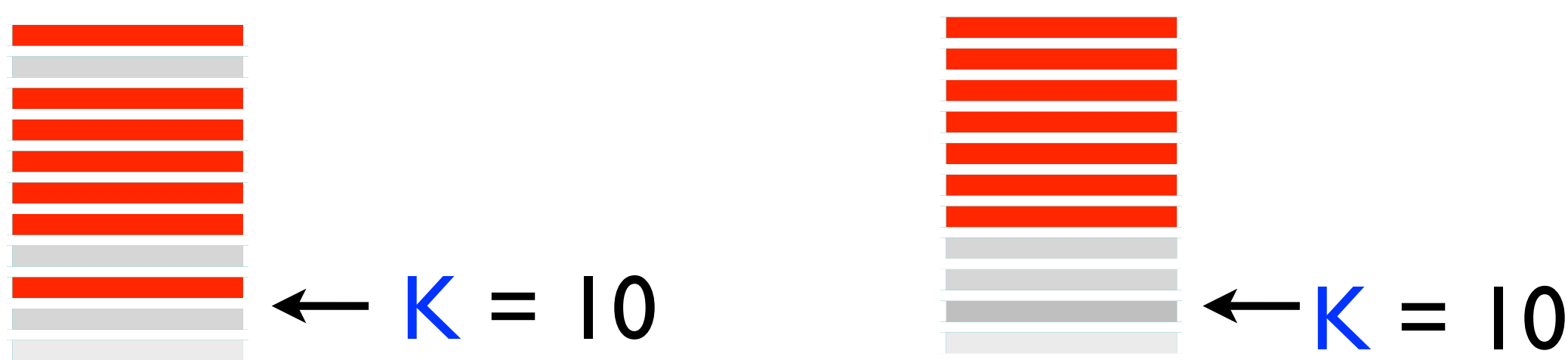


\section{Ranked Retrieval precision and recall}

- The ranking of documents within the top $\mathrm{K}$ is inconsequential

- If we don't know what value of $K$ to chose, we can compute and report several:P/R@\{1,5,10,20\}

- There are evaluation metrics that do not require choosing $\mathrm{K}$ (as we will see)

- One advantage of P/R@K, however, is that they are easy to interpret 


\section{Ranked Retrieval \\ what do these statements mean?}

- As with most metrics, experimenters report average values (averaged across evaluation queries)

- System A obtains an average P@I0 of 0.50

- System A obtains an average P@I0 of 0.10

- System A obtains an average P@I of 0.50

- System A obtains an average P@20 of 0.20 


\section{Ranked Retrieval comparing systems}

- Good practice: always ask yourself "Are users likely to notice?"

- System A obtains an average P@I of 0.10

- System B obtains an average P@I of 0.20

- This is a $100 \%$ improvement.

- Are user's likely to notice? 


\section{Ranked Retrieval comparing systems}

- Good practice: always ask yourself "Are users likely to notice?"

- System A obtains an average P@I of 0.05

- System B obtains an average P@। of 0.10

- This is a $100 \%$ improvement.

- Are user's likely to notice? 


\section{Ranked Retrieval P/R@K}

- Advantages:

- easy to compute

- easy to interpret

- Disadvantages:

- the value of $K$ has a huge impact on the metric

- the ranking above $\mathrm{K}$ is inconsequential

- how do we pick K? 


\section{Ranked Retrieval}

motivation: average precision

- Ideally, we want the system to achieve high precision for varying values of $\mathrm{K}$

- The metric average precision accounts for precision and recall without having to set $\mathrm{K}$ 


\section{Ranked Retrieval}

average precision

1. Go down the ranking one-rank-at-a-time

2. If the document at rank $\mathrm{K}$ is relevant, measure $\mathrm{P@K}$

- proportion of top-K documents that are relevant

3. Finally, take the average of all P@K values

- the number of $\mathrm{P@K}$ values will equal the number of relevant documents 


\section{Ranked Retrieval}

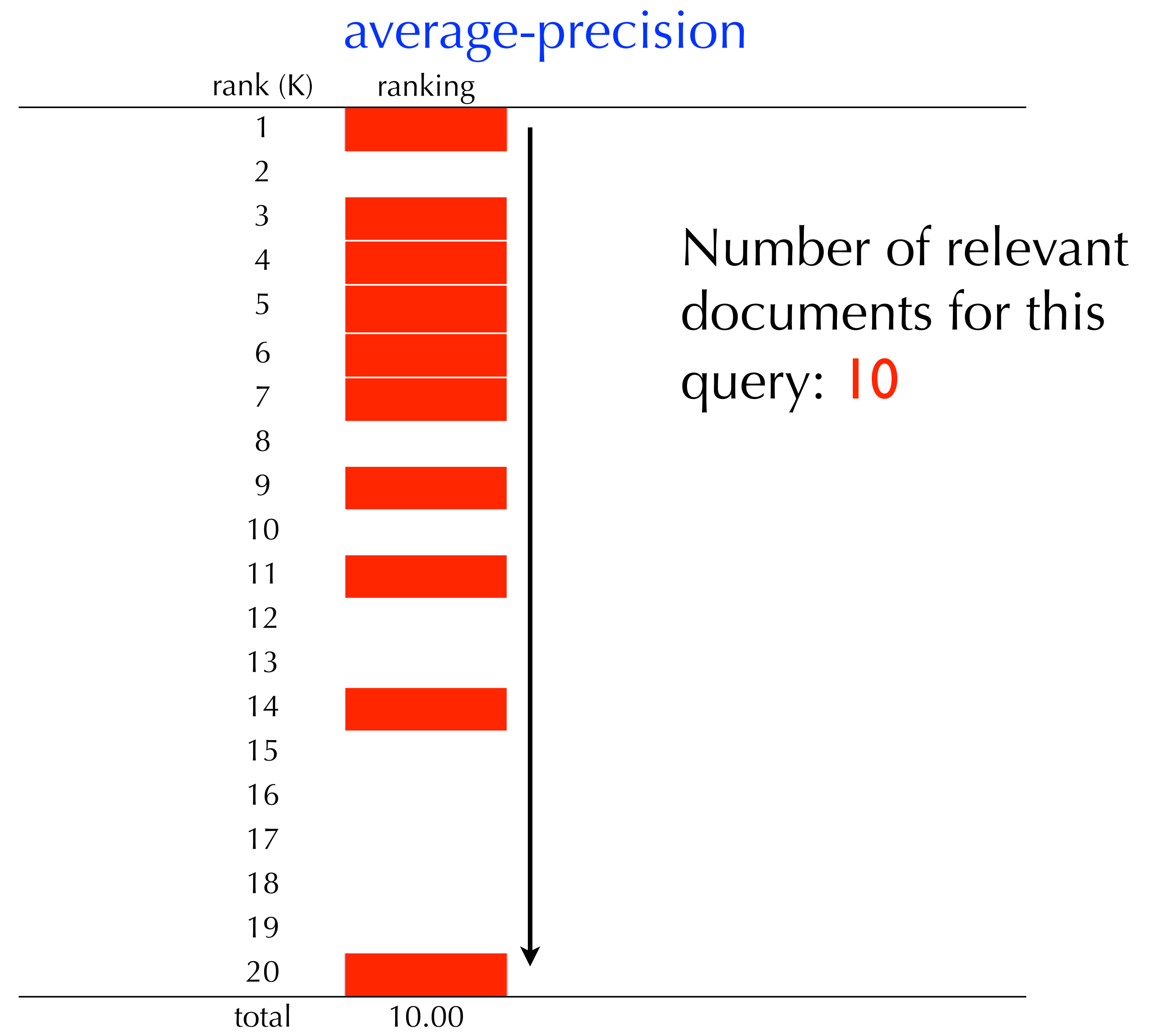




\section{Ranked Retrieval}

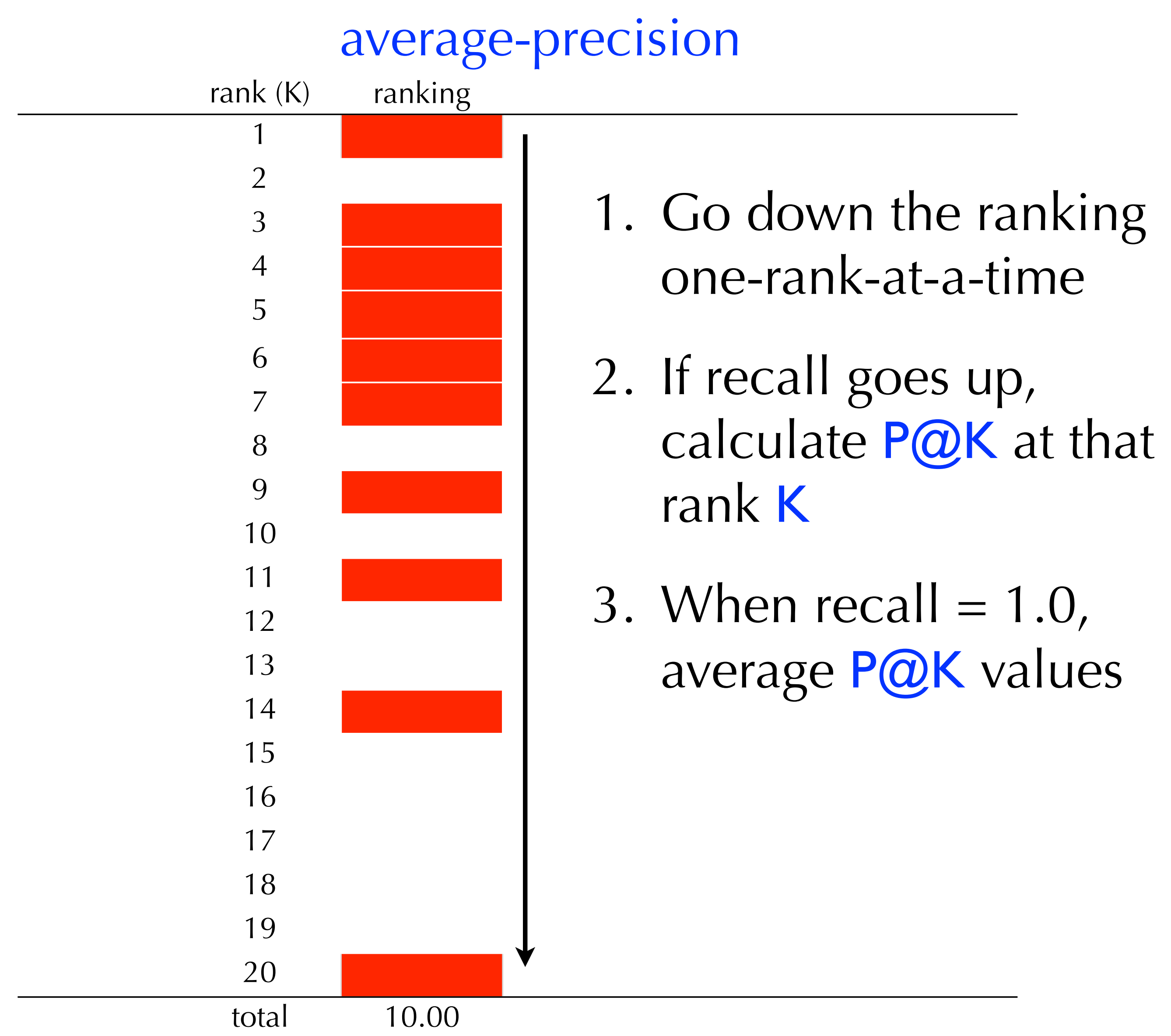




\section{Ranked Retrieval}

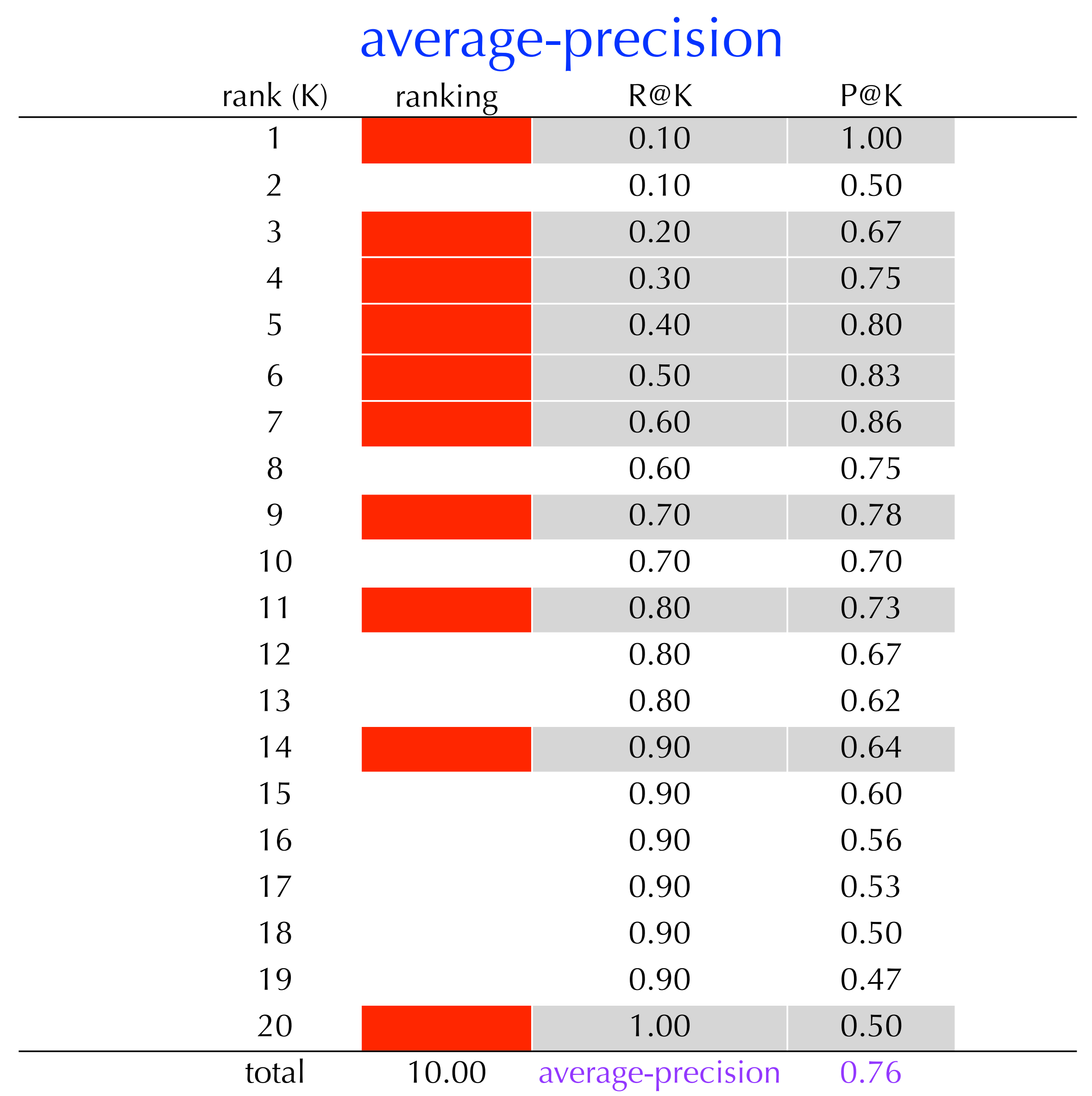




\section{Ranked Retrieval}

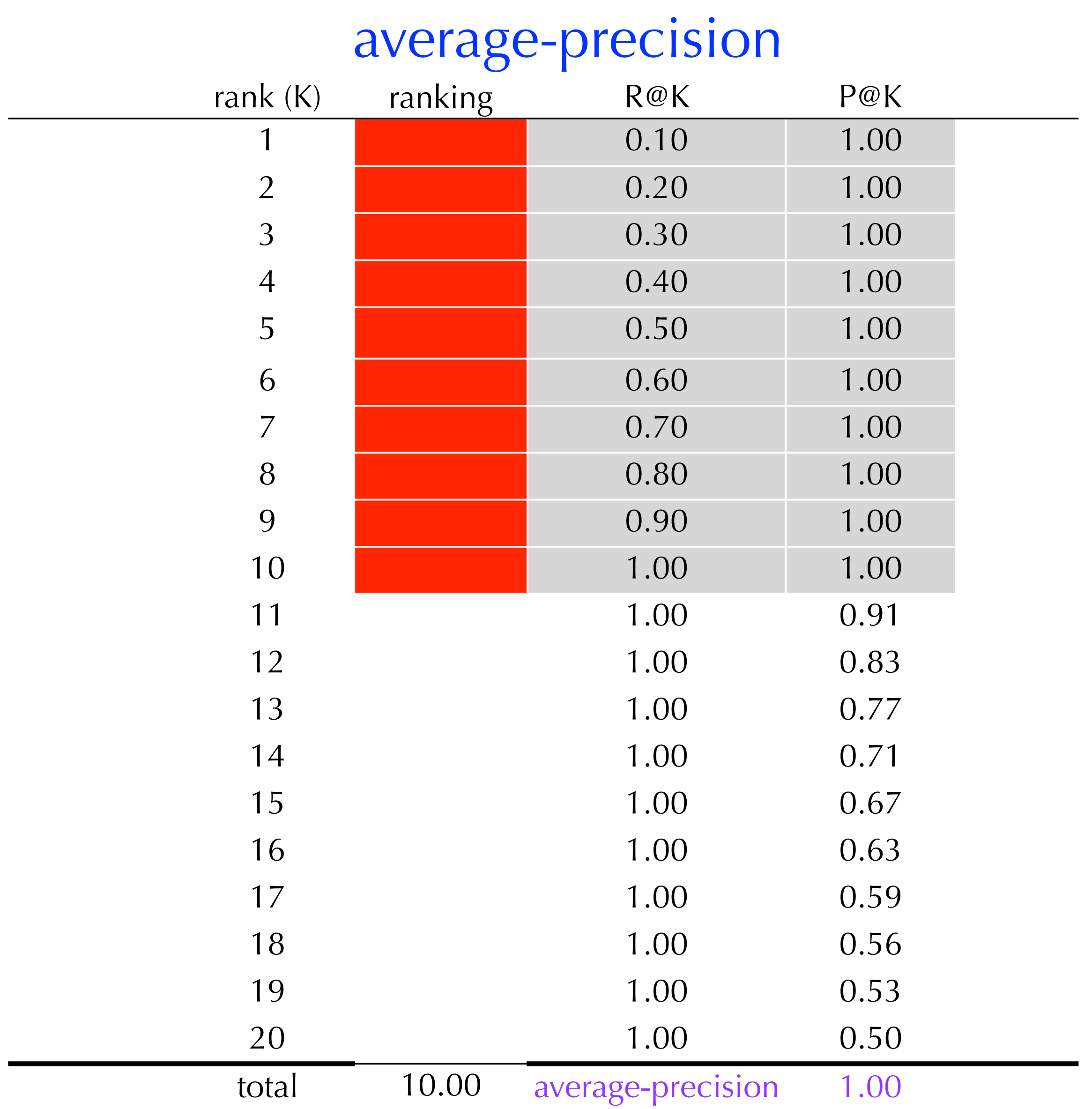




\section{Ranked Retrieval}

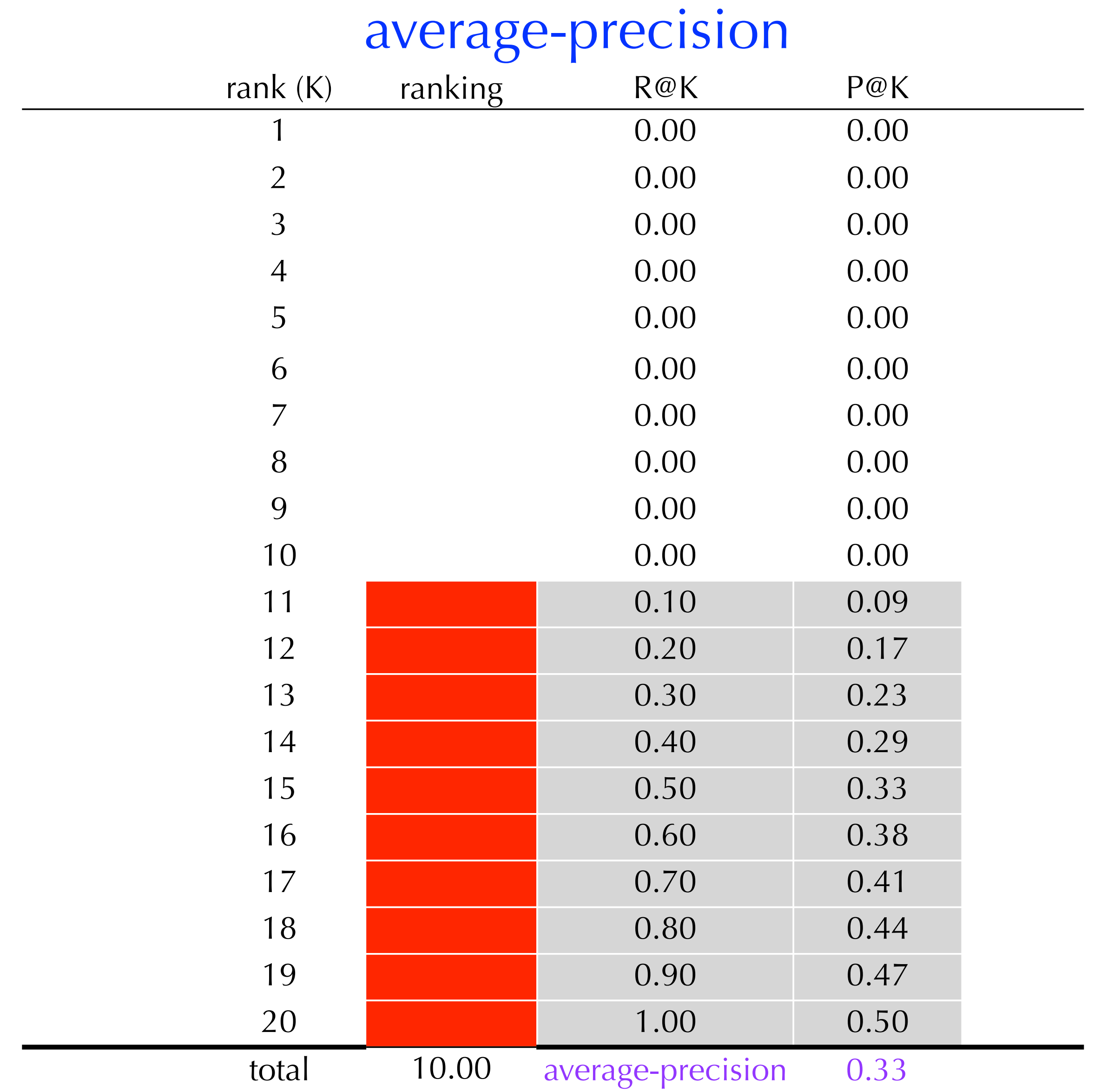




\section{Ranked Retrieval}

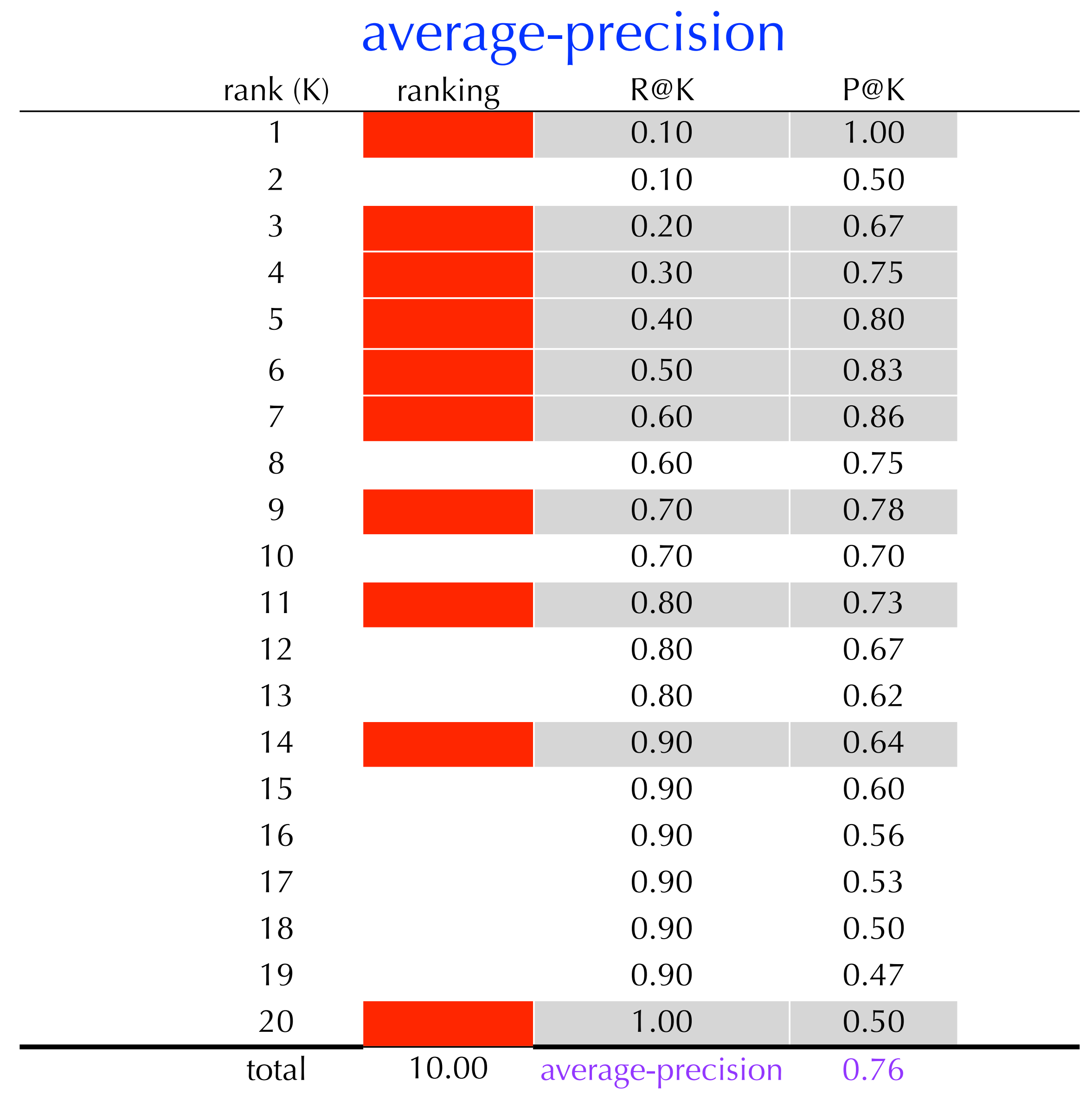




\section{Ranked Retrieval}

\begin{tabular}{|c|c|c|c|c|}
\hline & & verag & $\mathrm{ecis}$ & \\
\hline & $\operatorname{rank}(\mathrm{K})$ & ranking & $\mathrm{R} @ \mathrm{~K}$ & $\mathrm{P} @ K$ \\
\hline & 1 & & 0.10 & 1.00 \\
\hline swapped & 2 & & 0.20 & 1.00 \\
\hline anke? & 3 & & 0.20 & 0.67 \\
\hline anks 2 and 5 & 4 & & 0.30 & 0.75 \\
\hline & 5 & & 0.40 & 0.80 \\
\hline & 6 & & 0.50 & 0.83 \\
\hline & 7 & & 0.60 & 0.86 \\
\hline & 8 & & 0.60 & 0.75 \\
\hline & 9 & & 0.70 & 0.78 \\
\hline & 10 & & 0.70 & 0.70 \\
\hline & 11 & & 0.80 & 0.73 \\
\hline & 12 & & 0.80 & 0.67 \\
\hline & 13 & & 0.80 & 0.62 \\
\hline & 14 & & 0.90 & 0.64 \\
\hline & 15 & & 0.90 & 0.60 \\
\hline & 16 & & 0.90 & 0.56 \\
\hline & 17 & & 0.90 & 0.53 \\
\hline & 18 & & 0.90 & 0.50 \\
\hline & 19 & & 0.90 & 0.47 \\
\hline & 20 & & 1.00 & 0.50 \\
\hline & total & 10.00 & ge-pres & 0.79 \\
\hline
\end{tabular}




\section{Ranked Retrieval}

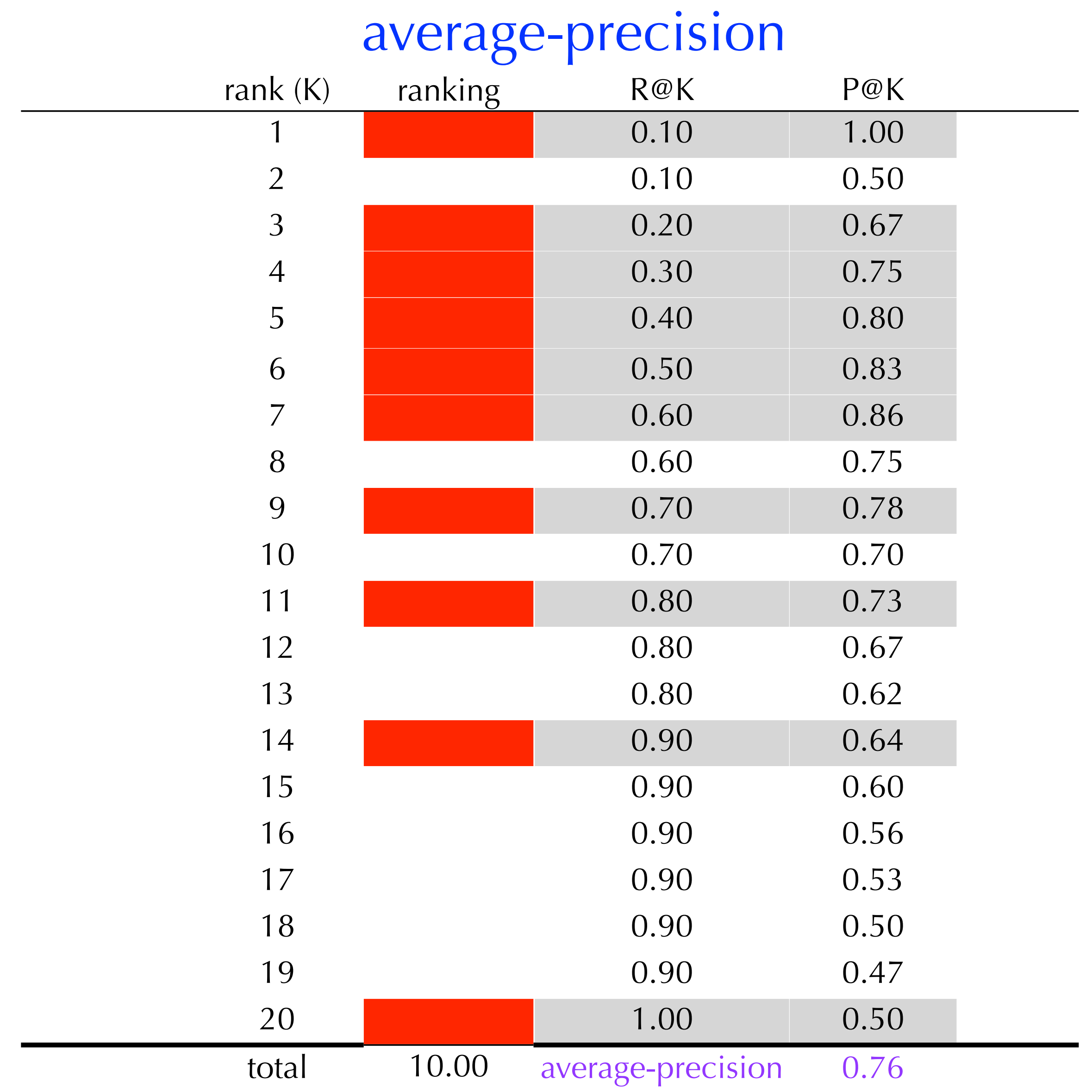




\section{Ranked Retrieval}

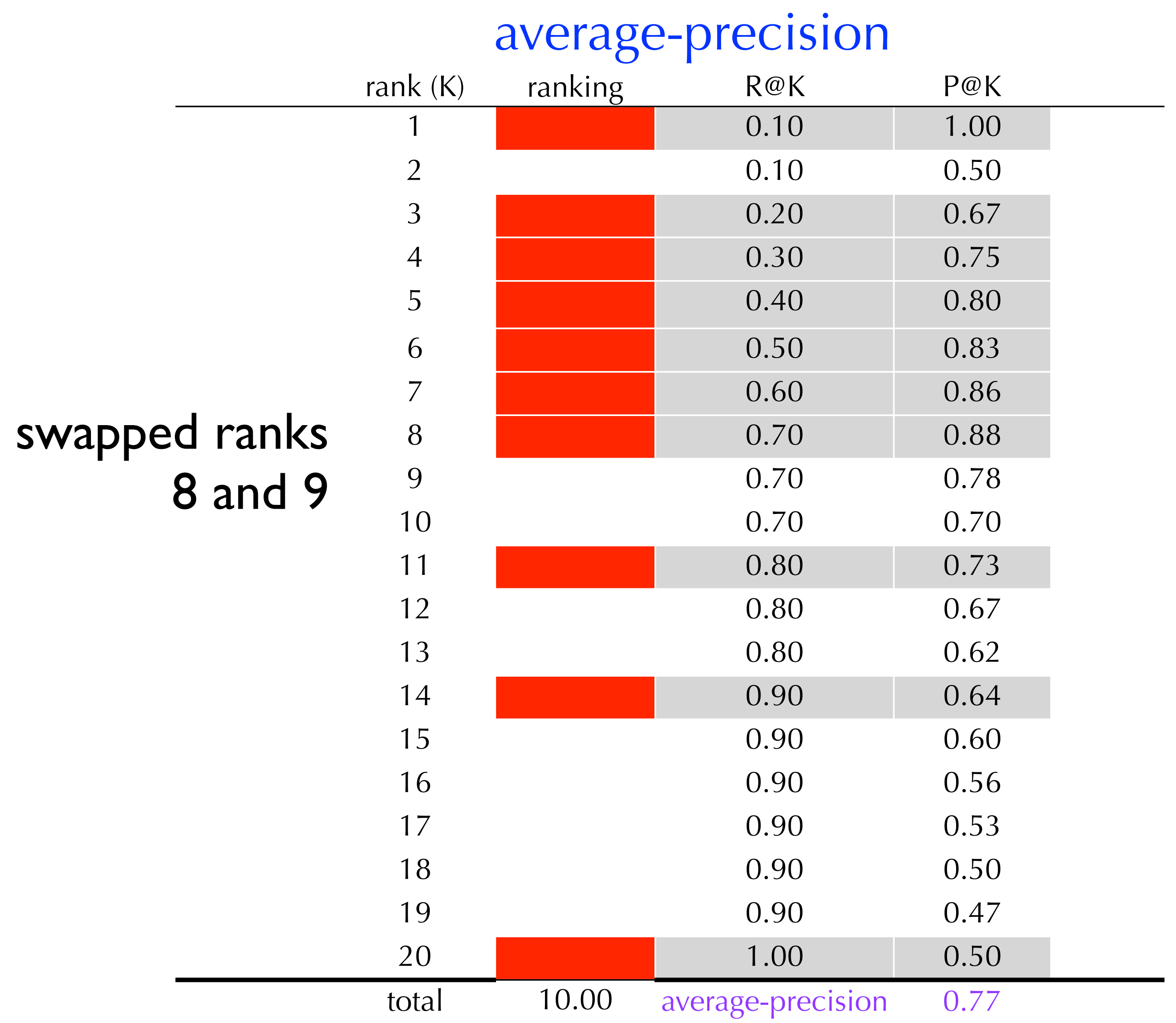




\section{Ranked Retrieval}

average precision

- Advantages:

- no need to choose $\mathrm{K}$

- accounts for both precision and recall

- ranking mistakes at the top of the ranking are more influential

- ranking mistakes at the bottom of the ranking are still accounted for

- Disadvantages

- not quite as easy to interpret as P/R@K 


\section{Ranked Retrieval MAP: mean average precision}

- So far, we've talked about average precision for a single query

- Mean Average Precision (MAP): average precision averaged across a set of queries

- yes, confusing. but, better than calling it "average average precision"!

- one of the most common metrics in IR evaluation 


\section{Ranked Retrieval precision-recall curves}

- In some situations, we want to understand the trade-off between precision and recall

- A precision-recall (PR) curve expresses precision as a function of recall 


\section{Ranked Retrieval precision-recall curves: general idea}

- Different tasks require different levels of recall

- Sometimes, the user wants a few relevant documents

- Other times, the user wants most of them

- Suppose a user wants some level of recall $R$

- The goal for the system is to minimize the number of false negatives the user must look at in order to achieve a level of recall $R$ 


\section{Ranked Retrieval precision-recall curves: general idea}

- False negative error: not retrieving a relevant document

- false negative errors affects recall

- False positive errors: retrieving a non-relevant document

- false positives errors affects precision

- If a user wants to avoid a certain level of falsenegatives, what is the level of false-positives he/she must filter through? 


\section{Ranked Retrieval precision-recall curves}

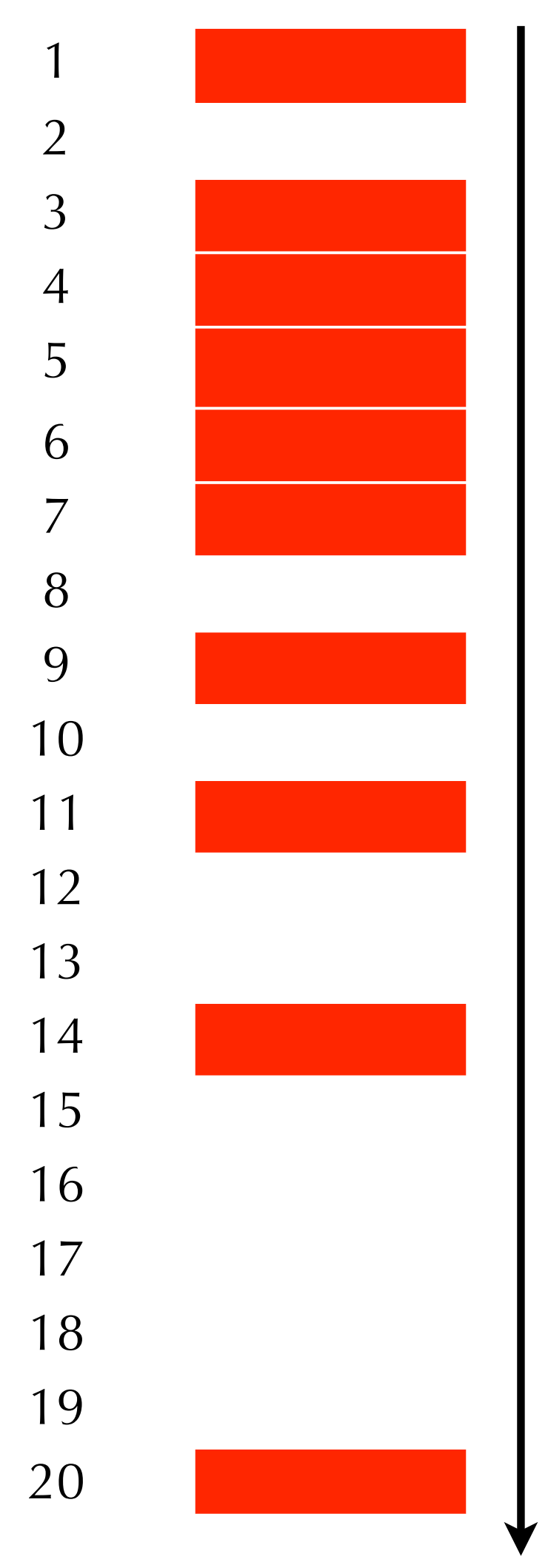

- Assume 10 relevant documents for this query

- Suppose the user wants $R=(\mathrm{I} / \mathrm{I} 0)$

- What level of precision will the user observe? 


\section{Ranked Retrieval precision-recall curves}

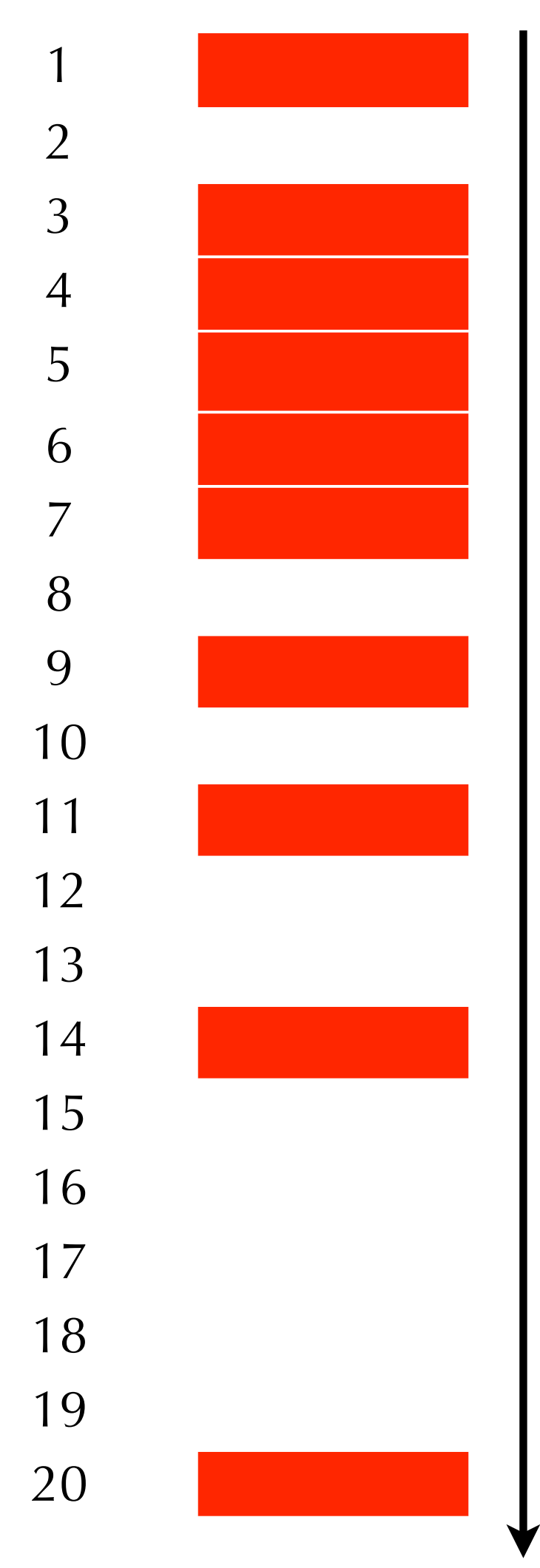

- Assume 10 relevant documents for this query

- Suppose the user wants $R=(2 / 10)$

- What level of precision will the user observe? 


\section{Ranked Retrieval precision-recall curves}

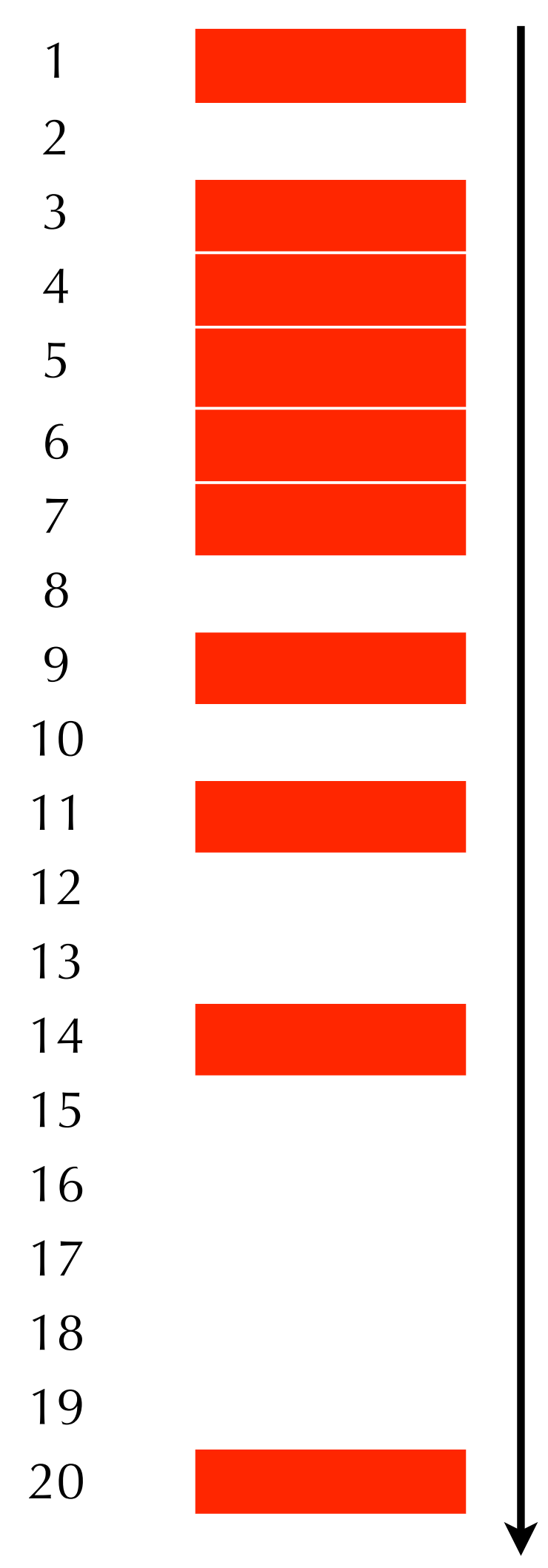

- Assume 10 relevant documents for this query

- Suppose the user wants $R=(10 / 10)$

- What level of precision will the user observe? 


\section{Ranked Retrieval}

\section{precision-recall curves}

\begin{tabular}{c|c|c|c} 
rank $(\mathrm{K})$ & ranking & R@K & P@K \\
\hline 1 & & 0.10 & 1.00 \\
2 & 0.10 & 0.50 \\
3 & 0.20 & 0.67 \\
4 & & 0.30 & 0.75 \\
5 & 0.40 & 0.80 \\
6 & 0.50 & 0.83 \\
7 & 0.60 & 0.86 \\
8 & 0.60 & 0.75 \\
9 & 0.70 & 0.78 \\
10 & 0.70 & 0.70 \\
11 & 0.80 & 0.73 \\
12 & 0.80 & 0.67 \\
13 & 0.80 & 0.62 \\
14 & 0.90 & 0.64 \\
15 & 0.90 & 0.60 \\
16 & 0.90 & 0.56 \\
17 & 0.90 & 0.53 \\
18 & 0.90 & 0.50 \\
19 & 0.90 & 0.47 \\
20 & 1.00 & 0.50 \\
\hline
\end{tabular}




\section{Ranked Retrieval precision-recall curves}

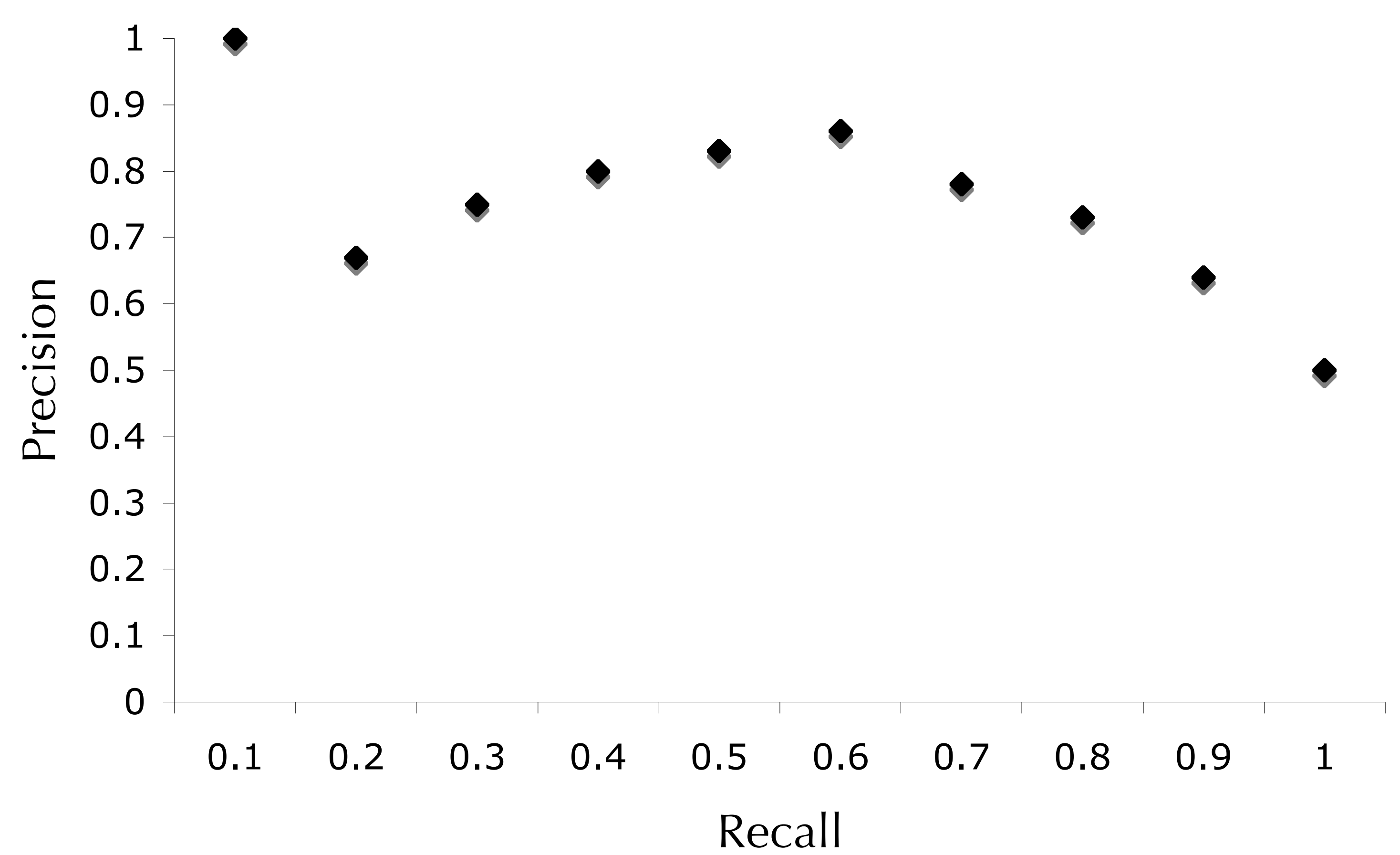




\section{Ranked Retrieval precision-recall curves}

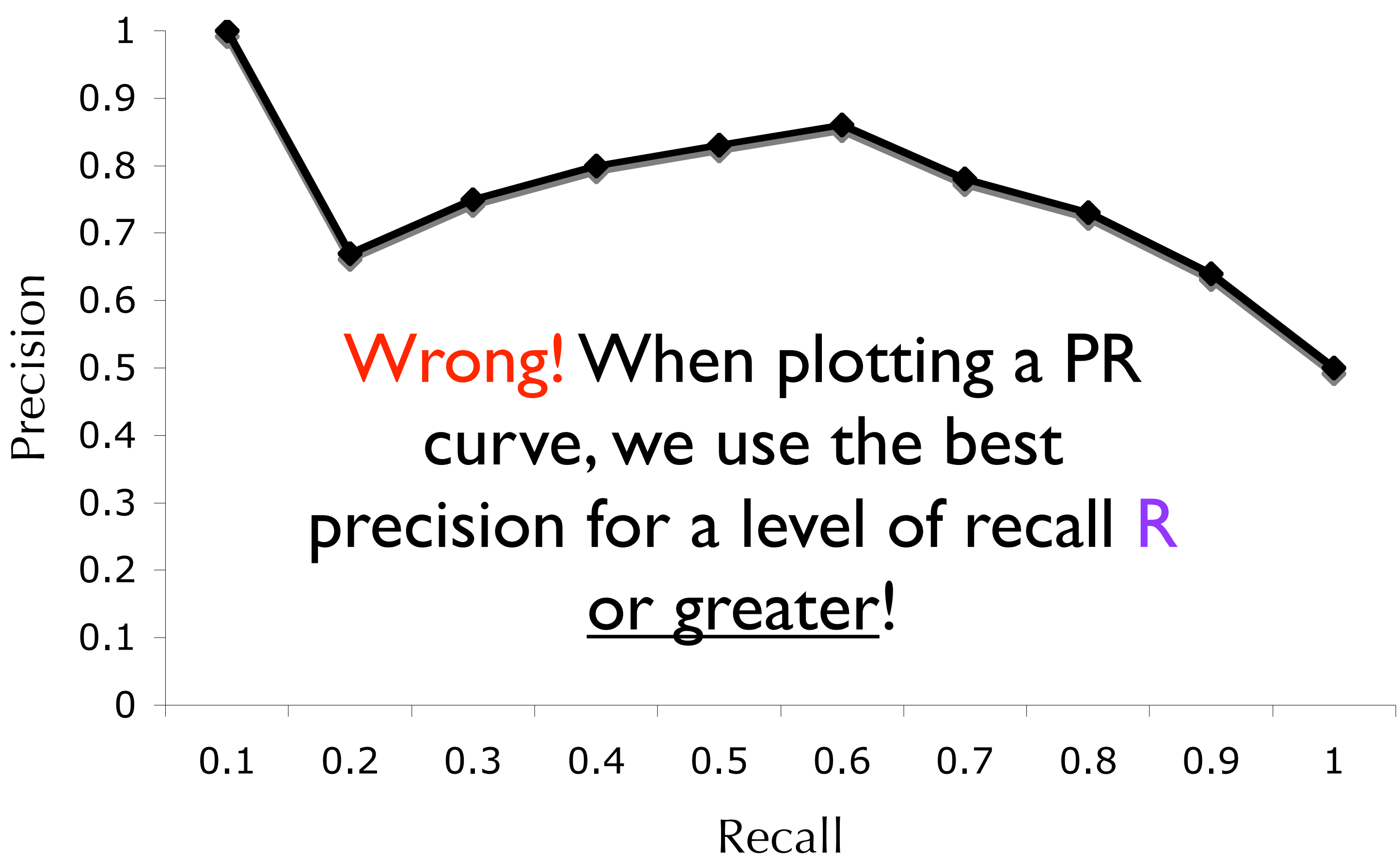




\section{Ranked Retrieval precision-recall curves}

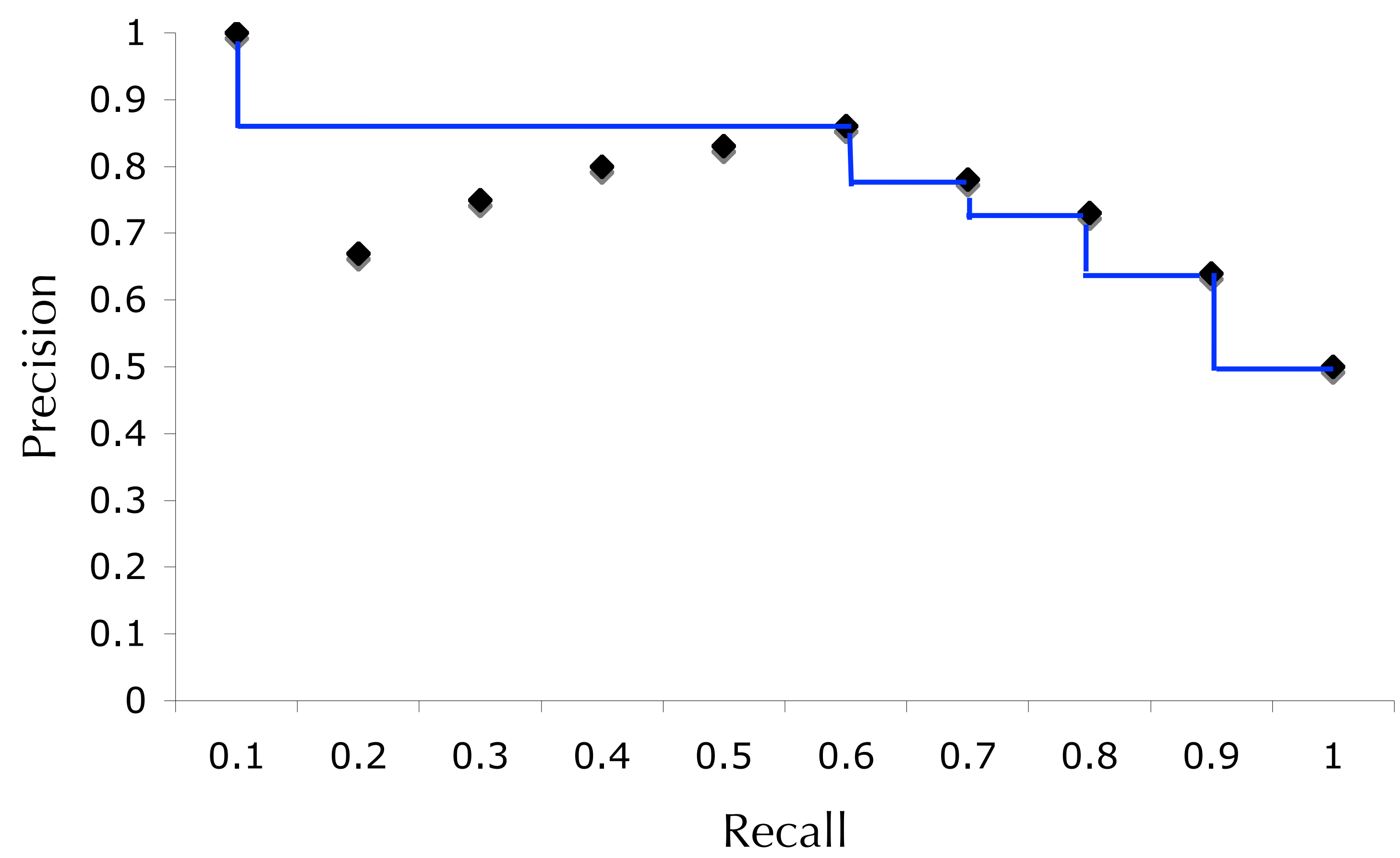




\section{Ranked Retrieval precision-recall curves}

- For a single query, a PR curve looks like a step-function

- For multiple queries, we can average these curves

- Average the precision values for different values of recall (e.g., from 0.01 to 1.0 in increments of 0.01 )

- This forms a smoother function 


\section{Ranked Retrieval precision-recall curves}

- PR curves can be averaged across multiple queries

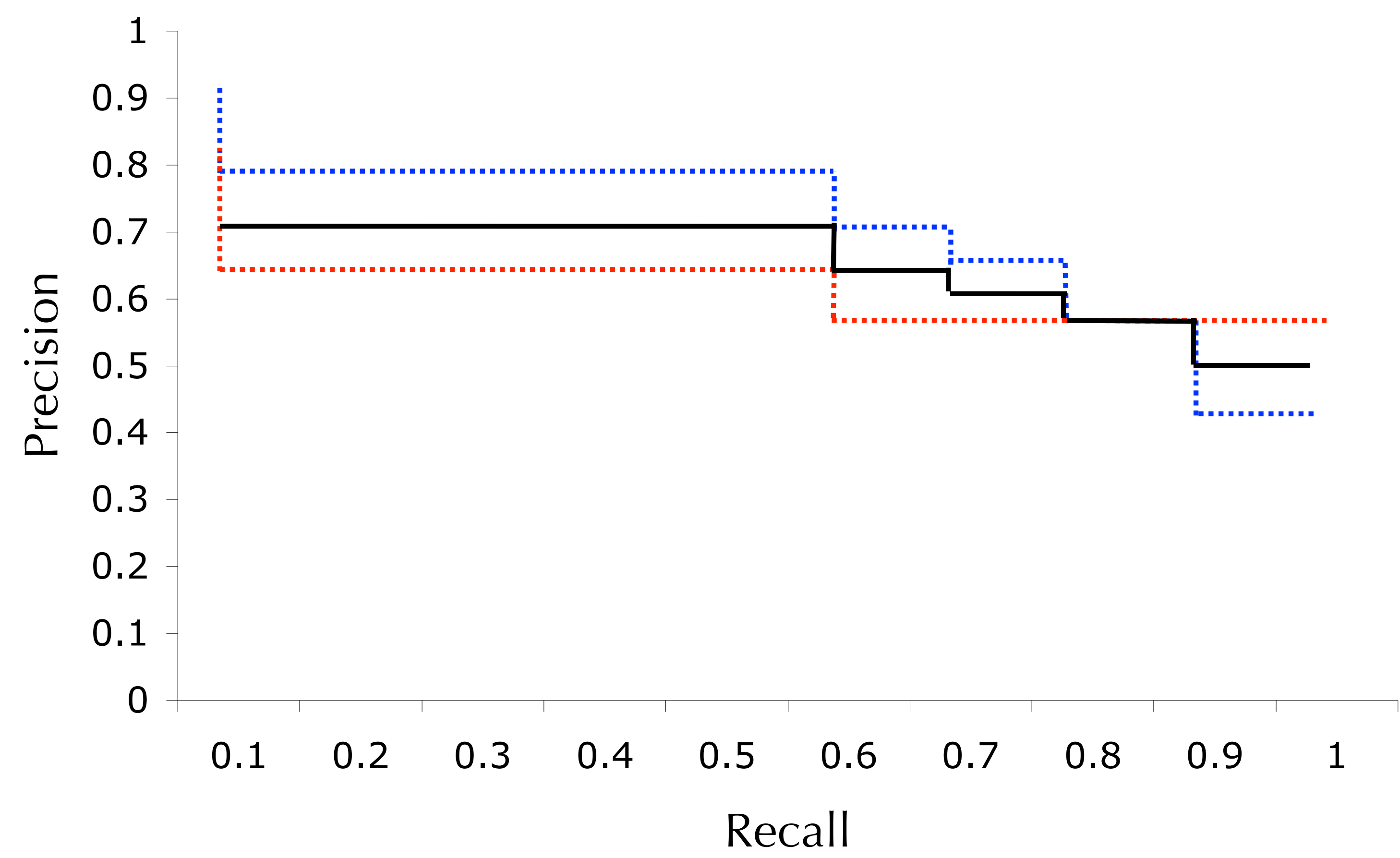




\section{Ranked Retrieval precision-recall curves}

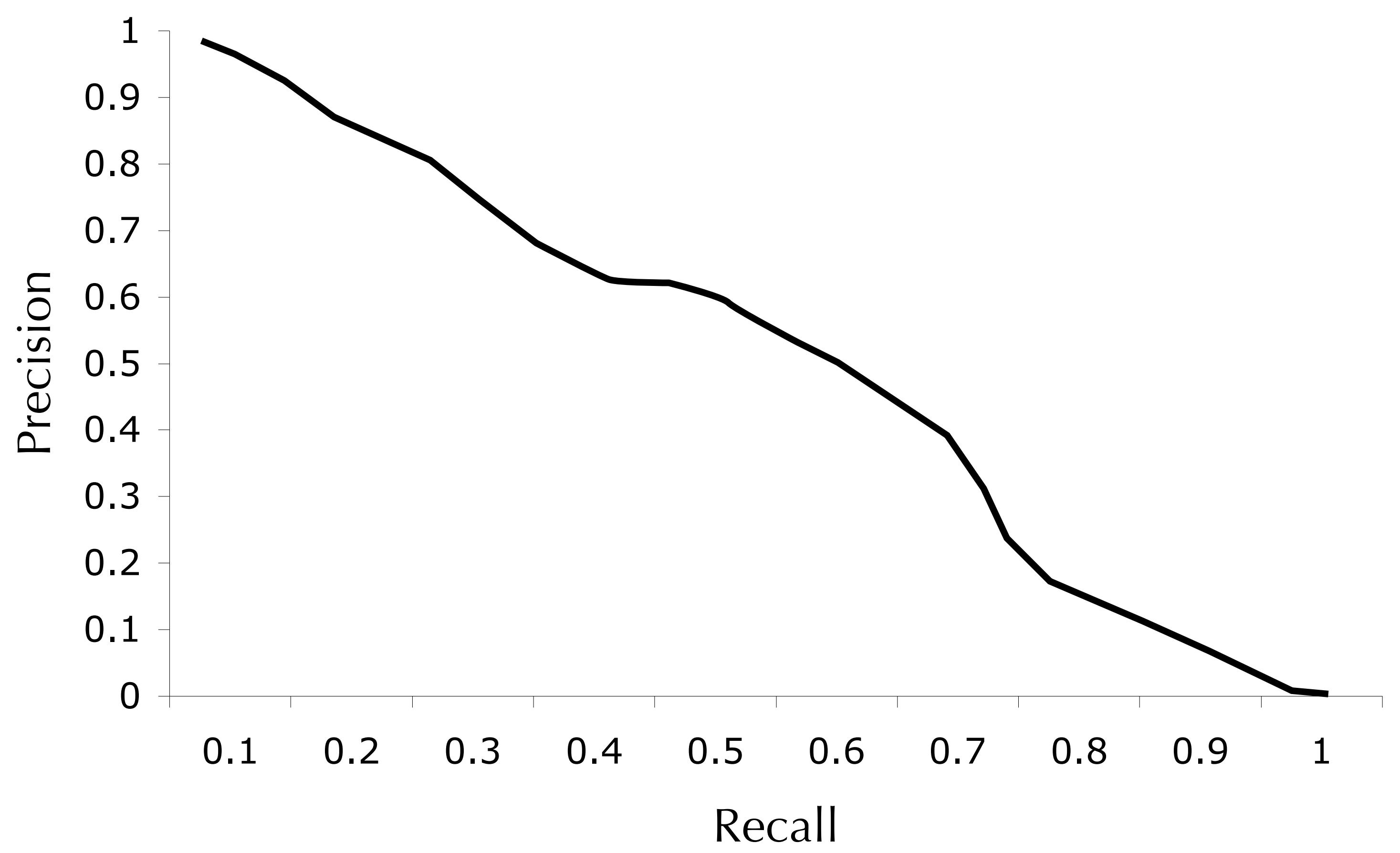




\section{Ranked Retrieval precision-recall curves}

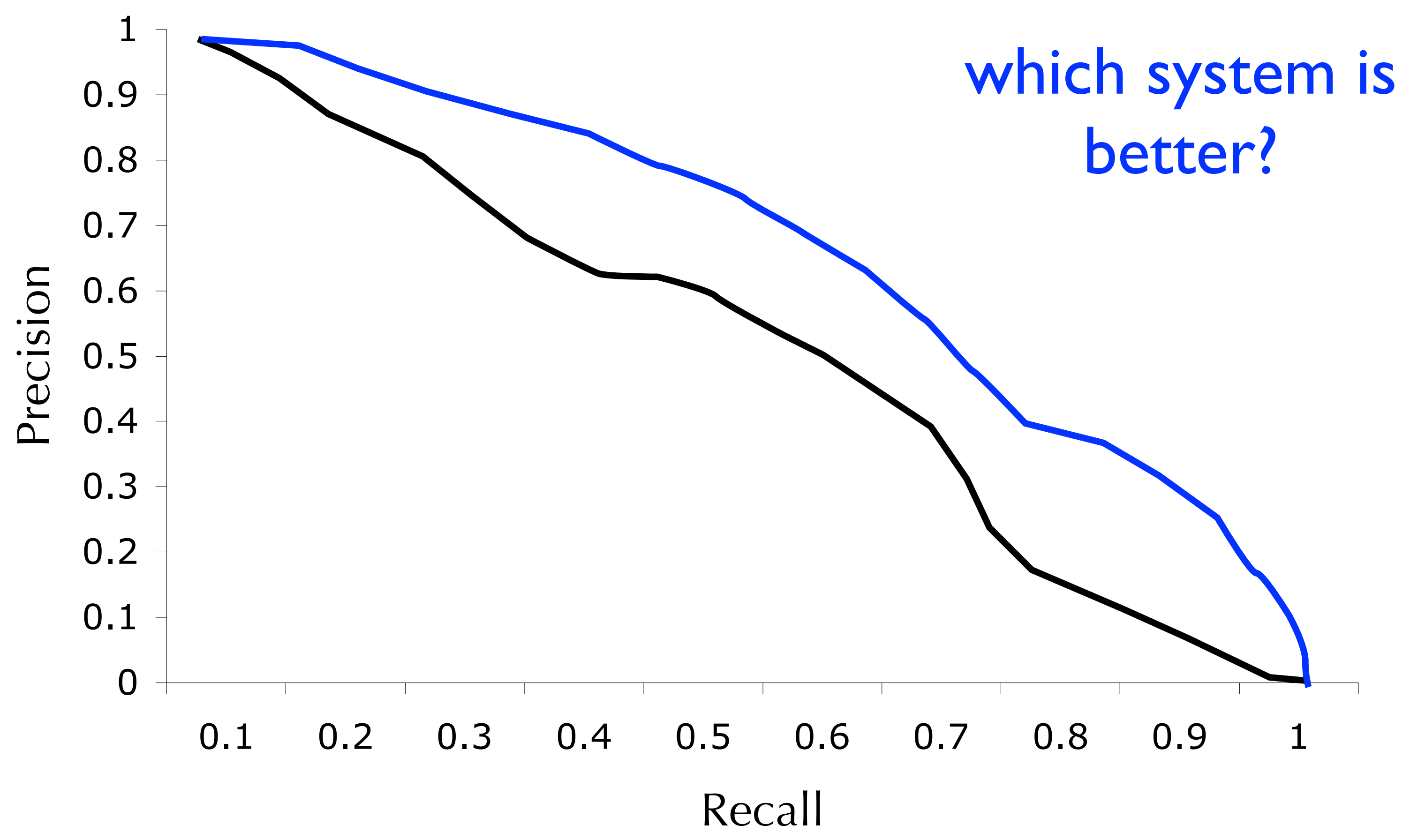




\section{Ranked Retrieval precision-recall curves}

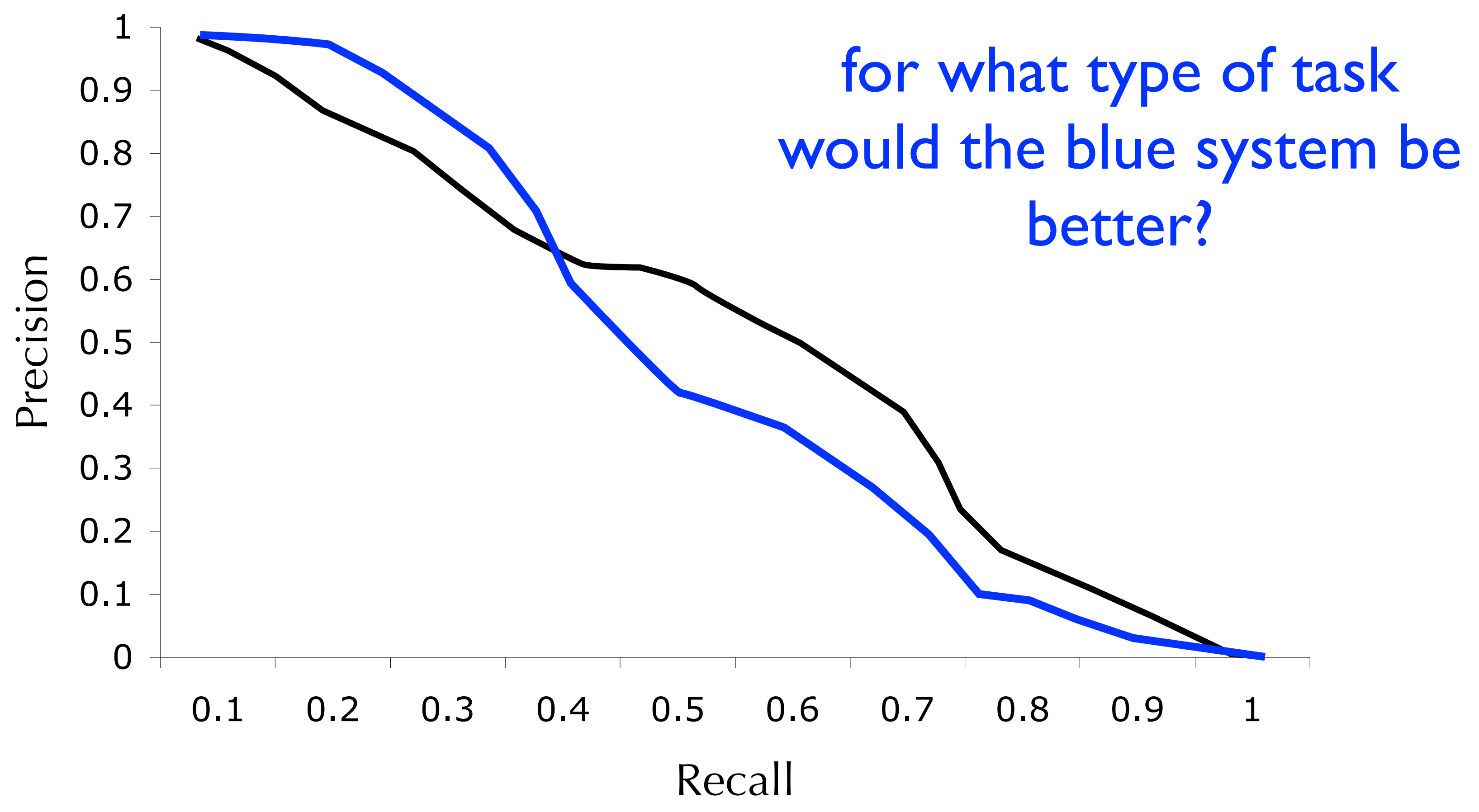




\section{Ranked Retrieval precision-recall curves}

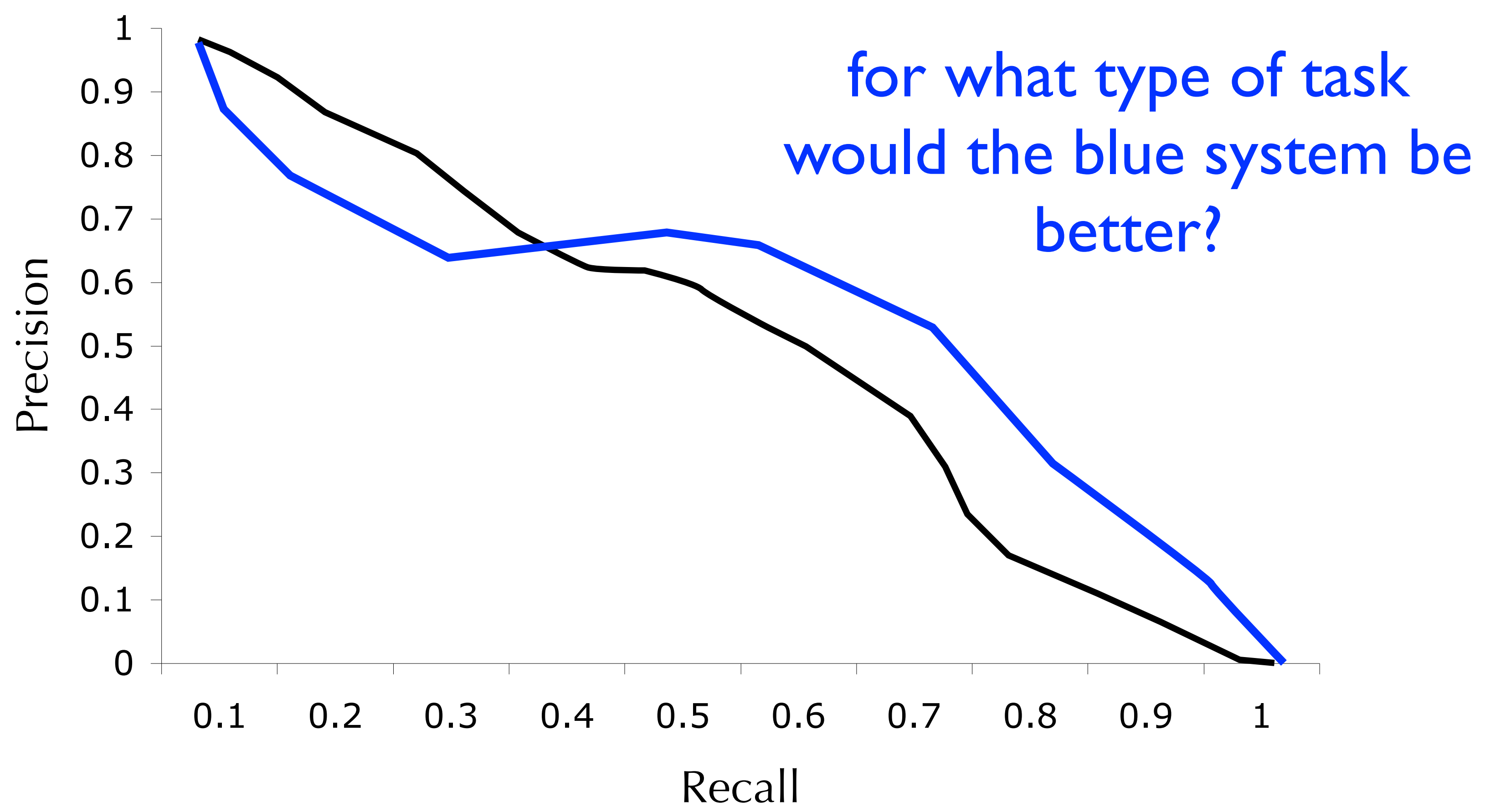




\section{Ranked Retrieval discounted-cumulative gain}

- In some retrieval tasks, we really want to focus on precision at the top of the ranking

- A classic example is web-search!

- users rarely care about recall

- users rarely navigate beyond the first page of results

- users may not even look at results below the "fold"

- Are any of the metrics we've seen so far appropriate for web-search? 


\section{Ranked Retrieval discounted-cumulative gain}

\section{CTR Curve}

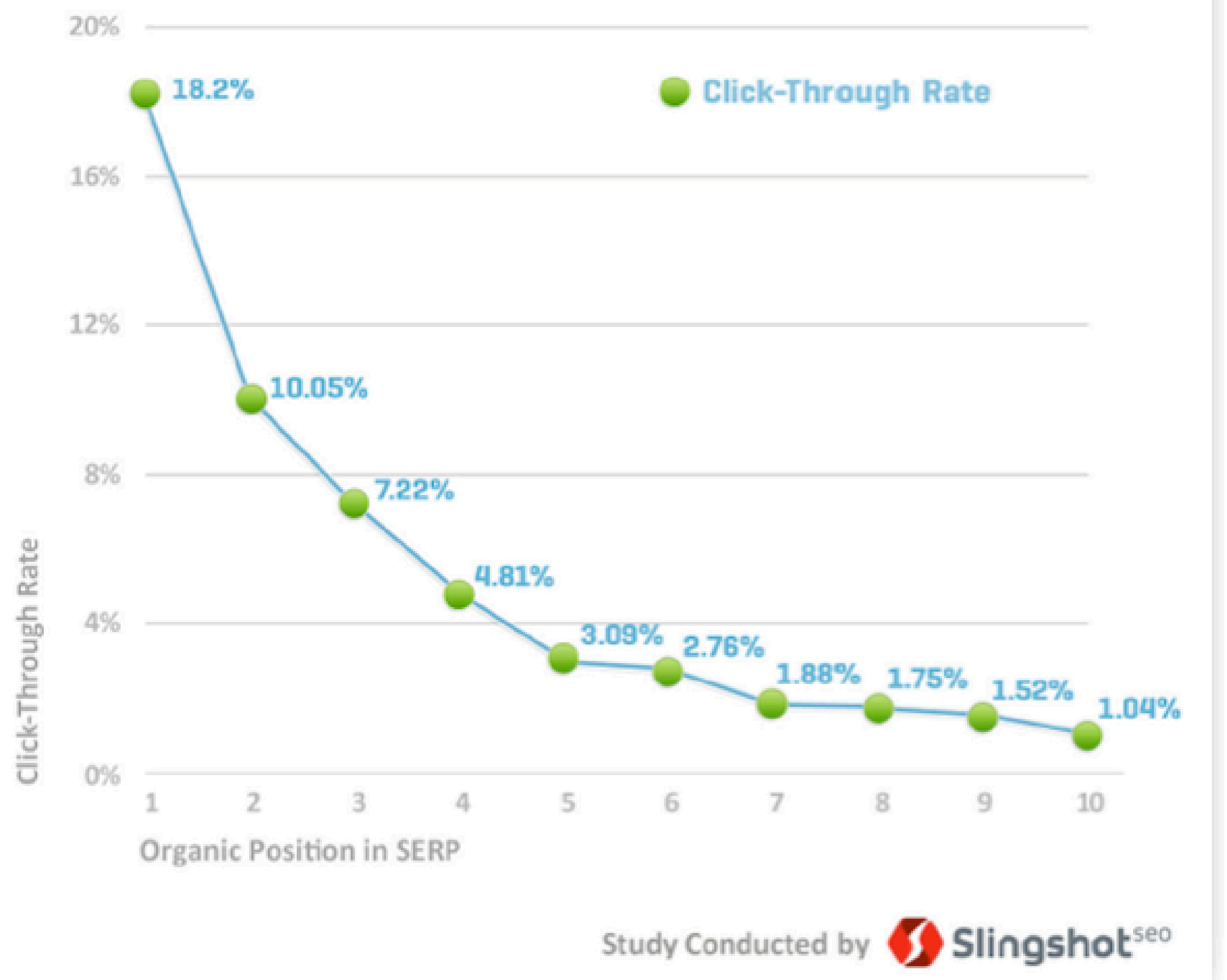




\section{Ranked Retrieval discounted-cumulative gain}

- We could potentially evaluate using P@K with several small values of $\mathrm{K}$

- But, this has some limitations

- What are they? 


\section{Ranked Retrieval discounted-cumulative gain}

- Which retrieval is better?
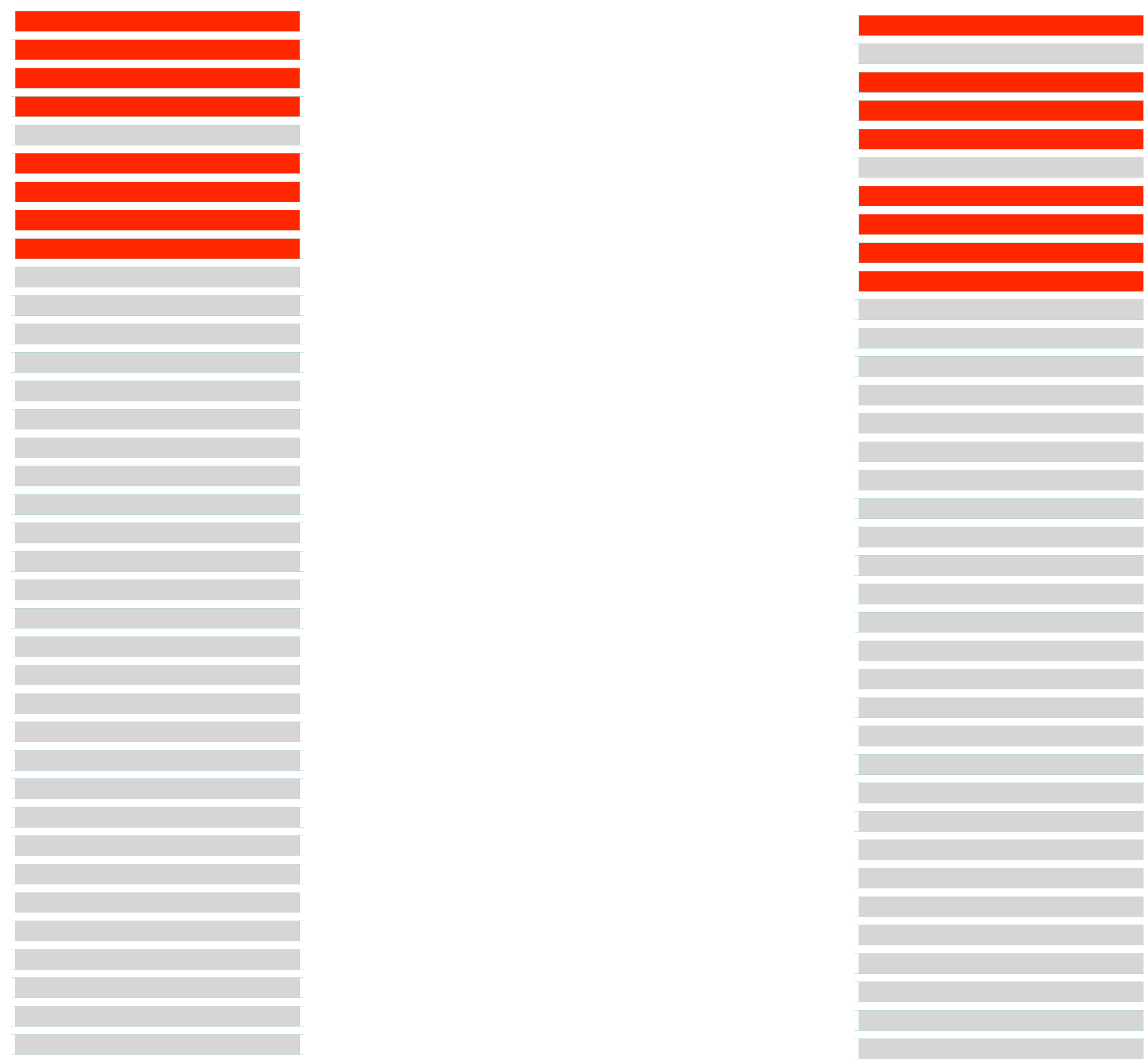


\section{Ranked Retrieval discounted-cumulative gain}

- Evaluation based on P@K can be too coarse
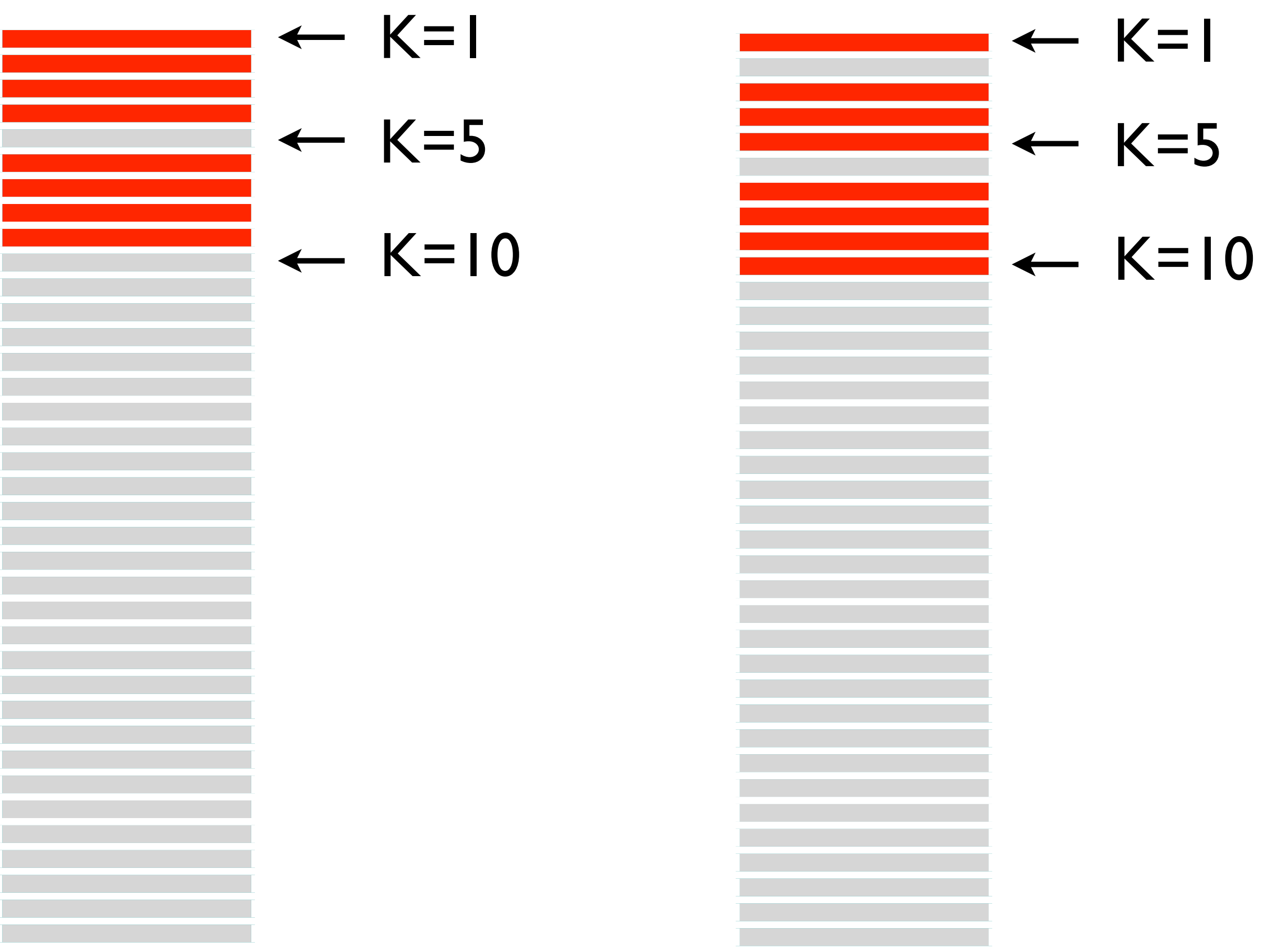


\section{Ranked Retrieval discounted-cumulative gain}

- $\mathrm{P} @ \mathrm{~K}$ (and all the metrics we've seen so far) assume binary relevance
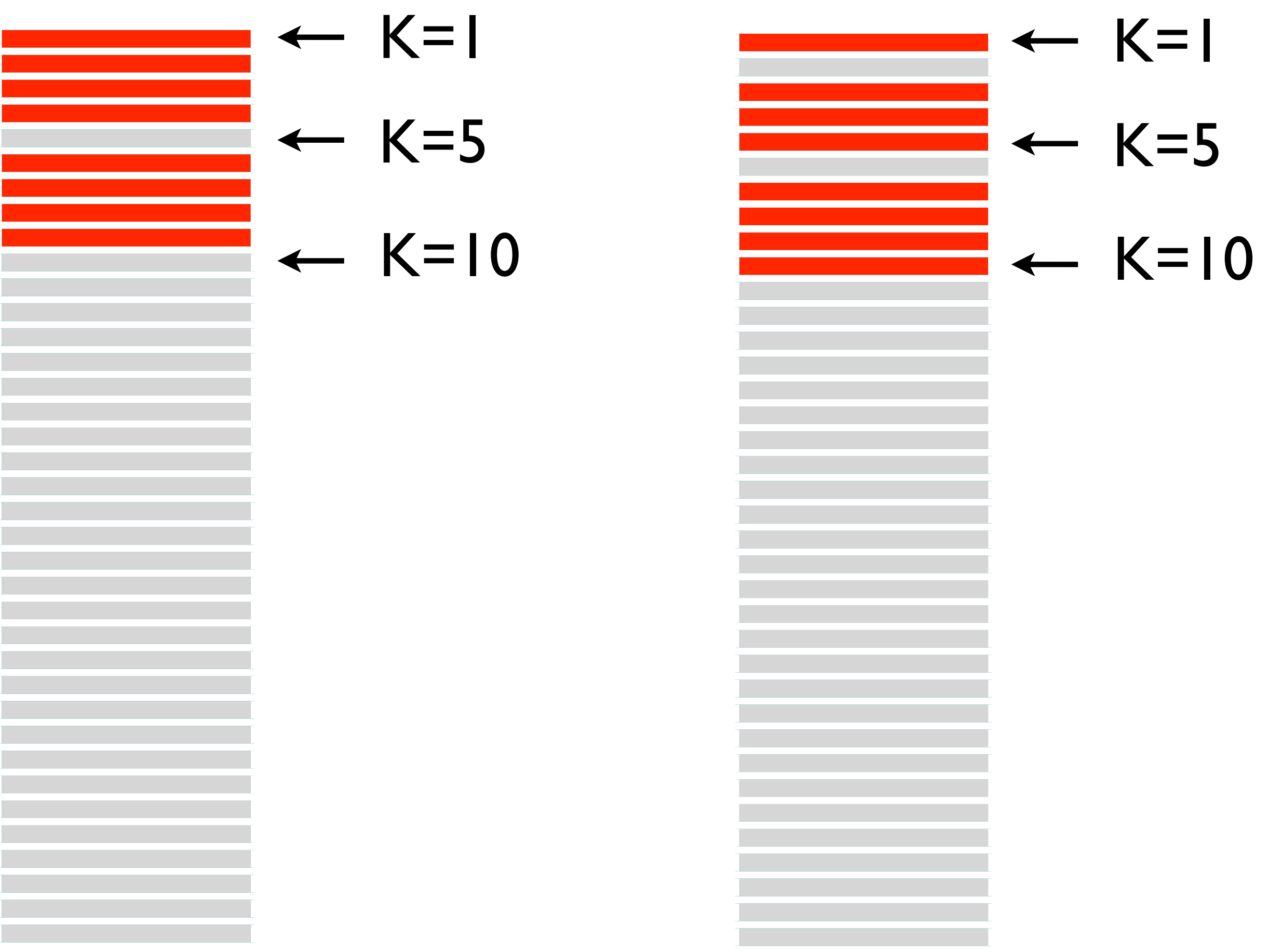


\section{Ranked Retrieval discounted-cumulative gain}

- DCG: discounted cumulative gain

- Assumptions:

- There are more than two levels of relevance (e.g., perfect, excellent, good, fair, bad)

- A relevant document's usefulness to a user decreases rapidly with rank (more rapidly than linearly) 


\section{Ranked Retrieval discounted-cumulative gain}

- Let $R E L_{i}$ be the relevance associated with the document at rank $i$

1 perfect $\rightarrow 4$

- excellent $\rightarrow 3$

, $\operatorname{good} \rightarrow 2$

- fair $\rightarrow 1$

- bad $\rightarrow 0$ 


\section{Ranked Retrieval discounted-cumulative gain}

- DCG: discounted cumulative gain

$$
D C G @ K=\sum_{i=1}^{K} \frac{R E L_{i}}{\log _{2}(\max (i, 2))}
$$




\section{Ranked Retrieval discounted-cumulative gain}

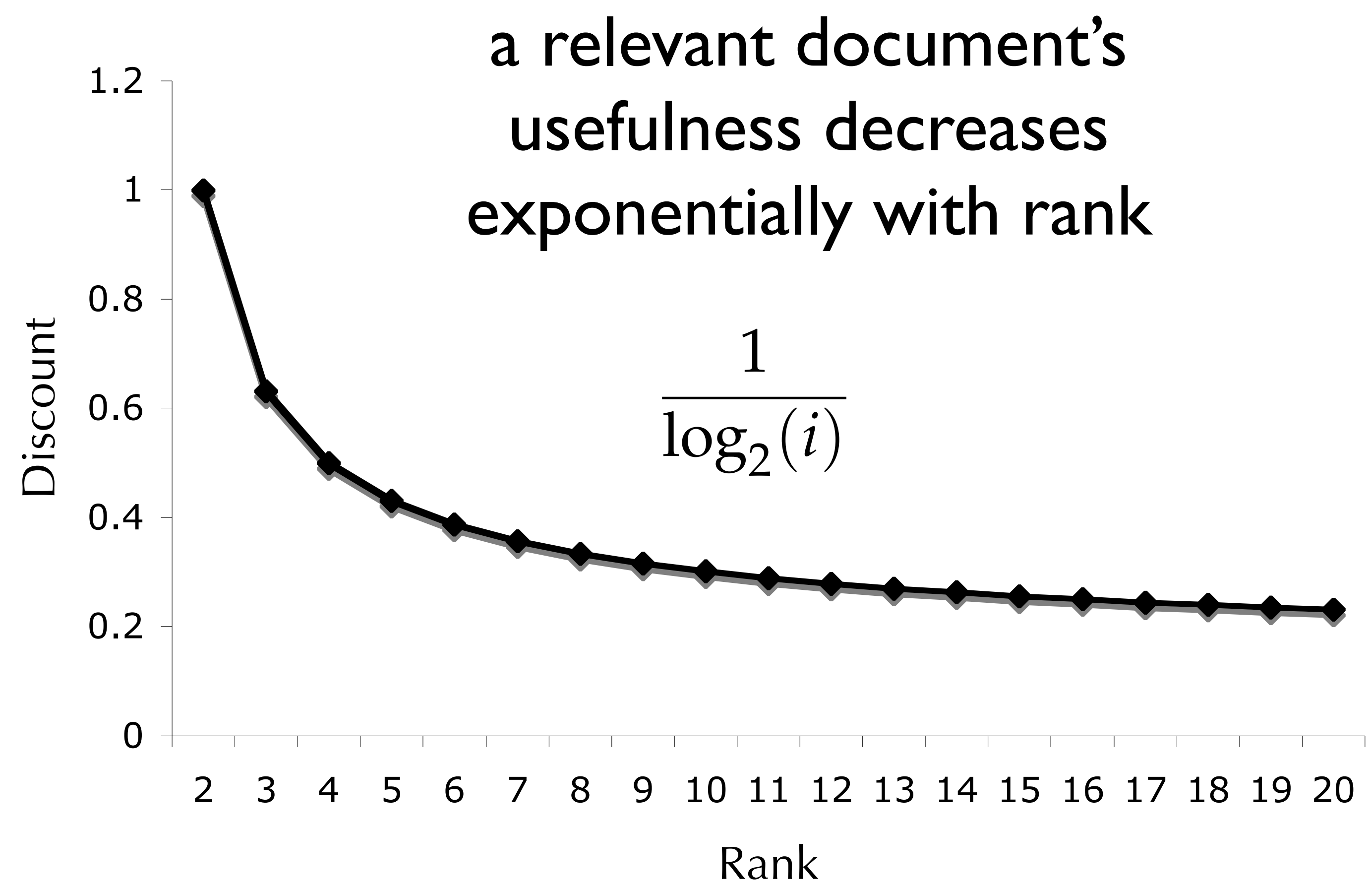




\section{Ranked Retrieval}

discounted-cumulative gain

$$
D C G @ K=\sum_{i=1}^{K} \frac{R E L_{i}}{\log _{2}(\max (i, 2))}
$$

\begin{tabular}{cc}
$\operatorname{rank}(\mathrm{i})$ & REL_i \\
\hline 1 & 4 \\
2 & 3 \\
3 & 4 \\
4 & 2 \\
5 & 0 \\
6 & 0 \\
7 & 0 \\
8 & 1 \\
9 & 1 \\
10 & 0
\end{tabular}

This is given!

the result at rank $I$ is perfect the result at rank 2 is excellent the result at rank 3 is perfect

the result at rank 10 is bad 
Ranked Retrieval

discounted-cumulative gain

$$
D C G @ K=\sum_{i=1}^{K} \frac{R E L_{i}}{\log _{2}(\max (i, 2))}
$$

\begin{tabular}{ccc} 
rank (i) & REL_i & discount factor \\
\hline 1 & 4 & 1.00 \\
2 & 3 & 1.00 \\
3 & 4 & 0.63 \\
4 & 2 & 0.50 \\
5 & 0 & 0.43 \\
6 & 0 & 0.39 \\
7 & 0 & 0.36 \\
8 & 1 & 0.33 \\
9 & 1 & 0.32 \\
10 & 0 & 0.30
\end{tabular}

Each rank is associated with a discount factor

$\frac{1}{\log _{2}(\max (i, 2))}$

rank $I$ is a special case! 
Ranked Retrieval

discounted-cumulative gain

$$
D C G @ K=\sum_{i=1}^{K} \frac{R E L_{i}}{\log _{2}(\max (i, 2))}
$$

\begin{tabular}{ccccl} 
rank (i) & REL_i & discount factor & gain & \\
\cline { 1 - 3 } 1 & 4 & 1.00 & 4.00 & \multirow{2}{*}{ multiply $R E L_{i}$} \\
2 & 3 & 1.00 & 3.00 & by the \\
3 & 4 & 0.63 & 2.52 & discount \\
4 & 2 & 0.50 & 1.00 & factor \\
5 & 0 & 0.43 & 0.00 & associated \\
6 & 0 & 0.39 & 0.00 & with the \\
7 & 0 & 0.36 & 0.00 & rank! \\
8 & 1 & 0.33 & 0.33 & \\
9 & 1 & 0.32 & 0.32 & \\
10 & 0 & 0.30 & 0.00 &
\end{tabular}


Ranked Retrieval

discounted-cumulative gain

$$
D C G @ K=\sum_{i=1}^{K} \frac{R E L_{i}}{\log _{2}(\max (i, 2))}
$$

\begin{tabular}{ccccc} 
rank (i) & REL_i & discount factor & gain & DCG_i \\
\hline 1 & 4 & 1.00 & 4.00 & 4.00 \\
2 & 3 & 1.00 & 3.00 & 7.00 \\
3 & 4 & 0.63 & 2.52 & 9.52 \\
4 & 2 & 0.50 & 1.00 & 10.52 \\
5 & 0 & 0.43 & 0.00 & 10.52 \\
6 & 0 & 0.39 & 0.00 & 10.52 \\
7 & 0 & 0.36 & 0.00 & 10.52 \\
8 & 1 & 0.33 & 0.33 & 10.86 \\
9 & 1 & 0.32 & 0.32 & 11.17 \\
10 & 0 & 0.30 & 0.00 & 11.17
\end{tabular}


Ranked Retrieval

discounted-cumulative gain

$$
D C G_{10}=11.17
$$

\begin{tabular}{ccccc} 
rank (i) & REL_i & discount factor & gain & DCG_i \\
\hline 1 & 4 & 1.00 & 4.00 & 4.00 \\
2 & 3 & 1.00 & 3.00 & 7.00 \\
3 & 4 & 0.63 & 2.52 & 9.52 \\
4 & 2 & 0.50 & 1.00 & 10.52 \\
5 & 0 & 0.43 & 0.00 & 10.52 \\
6 & 0 & 0.39 & 0.00 & 10.52 \\
7 & 0 & 0.36 & 0.00 & 10.52 \\
8 & 1 & 0.33 & 0.33 & 10.86 \\
9 & 1 & 0.32 & 0.32 & 11.17 \\
10 & 0 & 0.30 & 0.00 & 11.17
\end{tabular}


Ranked Retrieval

discounted-cumulative gain

$$
D C G_{10}=11.17
$$

\begin{tabular}{ccccc} 
rank (i) & REL_i & discount factor & gain & DCG_i \\
\hline 1 & 3 & 1.00 & 3.00 & 3.00 \\
2 & 3 & 1.00 & 3.00 & 6.00 \\
3 & 4 & 0.63 & 2.52 & 8.52 \\
4 & 2 & 0.50 & 1.00 & 9.52 \\
5 & 0 & 0.43 & 0.00 & 9.52 \\
6 & 0 & 0.39 & 0.00 & 9.52 \\
7 & 0 & 0.36 & 0.00 & 9.52 \\
8 & 1 & 0.33 & 0.33 & 9.86 \\
9 & 1 & 0.32 & 0.32 & 10.17 \\
10 & 0 & 0.30 & 0.00 & 10.17
\end{tabular}

changed top result from perfect instead of excellent 
Ranked Retrieval

discounted-cumulative gain

$$
D C G_{10}=11.17
$$

\begin{tabular}{ccccc} 
rank (i) & REL_i & discount factor & gain & DCG_i \\
\hline 1 & 4 & 1.00 & 4.00 & 4.00 \\
2 & 3 & 1.00 & 3.00 & 7.00 \\
3 & 4 & 0.63 & 2.52 & 9.52 \\
4 & 2 & 0.50 & 1.00 & 10.52 \\
5 & 0 & 0.43 & 0.00 & 10.52 \\
6 & 0 & 0.39 & 0.00 & 10.52 \\
7 & 0 & 0.36 & 0.00 & 10.52 \\
8 & 1 & 0.33 & 0.33 & 10.86 \\
9 & 1 & 0.32 & 0.32 & 11.17 \\
10 & 3 & 0.30 & 0.90 & 12.08
\end{tabular}

changed 10 th result from bad to excellent 


\section{Ranked Retrieval normalized discounted-cumulative gain}

- DCG is not 'bounded'

- In other words, it ranges from zero to .....

- Makes it problematic to average across queries

- NDCG: normalized discounted-cumulative gain

- "Normalized" is a fancy way of saying, we change it so that it ranges from 0 to 1 


\section{Ranked Retrieval normalized discounted-cumulative gain}

- NDCGi: normalized discounted-cumulative gain

- For a given query, measure $D_{C G}$

- Then, divide this $D_{C G} G_{i}$ value by the best possible DCG for that query

- Measure $\mathrm{DCG}_{\mathrm{i}}$ for the best possible ranking for a given value $\mathrm{i}$ 


\section{Ranked Retrieval normalized discounted-cumulative gain}

- Given: a query has two 4's, one 3, and the rest are 0's

- Question: What is the best possible ranking for $\mathrm{i}=1$

- All these are equally good:

- $4,4,3, \ldots$.

- $4,3,4, \ldots$

- $4,0,0, \ldots$

- ... anything with a 4 as the top-ranked result 


\section{Ranked Retrieval normalized discounted-cumulative gain}

- Given: the query has two 4's, one 3, and the rest are 0's

- Question: What is the best possible ranking for $\mathrm{i}=2$

- All these are equally good:

- $4,4,3, \ldots$.

- $4,4,0, \ldots$ 


\section{Ranked Retrieval normalized discounted-cumulative gain}

- Given: the query has two 4's, one 3, and the rest are 0's

- Question: What is the best possible ranking for $\mathrm{i}=3$

- All these are equally good:

- $4,4,3, \ldots$ 


\section{Ranked Retrieval normalized discounted-cumulative gain}

- NDCGi: normalized discounted-cumulative gain

- For a given query, measure $D_{C G}$

- Then, divide this $D_{C G} G_{i}$ value by the best possible DCG for that query

- Measure $\mathrm{DCG}_{\mathrm{i}}$ for the best possible ranking for a given value i 


\section{Metric Review}

- set-retrieval evaluation: we want to evaluate the set of documents retrieved by the system, without considering the ranking

- ranked-retrieval evaluation: we want to evaluate the ranking of documents returned by the system 


\section{Metric Review set-retrieval evaluation}

- precision: the proportion of retrieved documents that are relevant

- recall: the proportion of relevant documents that are retrieved

- $\mathrm{f}$-measure: harmonic-mean of precision and recall

- a difficult metric to "cheat" by getting very high precision and abysmal recall (and vice-versa) 


\section{Metric Review ranked-retrieval evaluation}

- $\mathrm{P} @ \mathrm{~K}:$ precision under the assumption that the top-K results is the 'set' retrieved

- $\mathrm{R} @ \mathrm{~K}$ : recall under the assumption that the top-K results is the 'set' retrieved

- average-precision: considers precision and recall and focuses mostly on the top results

- DCG: ignores recall, considers multiple levels of relevance, and focuses very must on the top ranks

- NDCG: trick to make DCG range between 0 and 1 


\section{Which Metric Would You Use?}

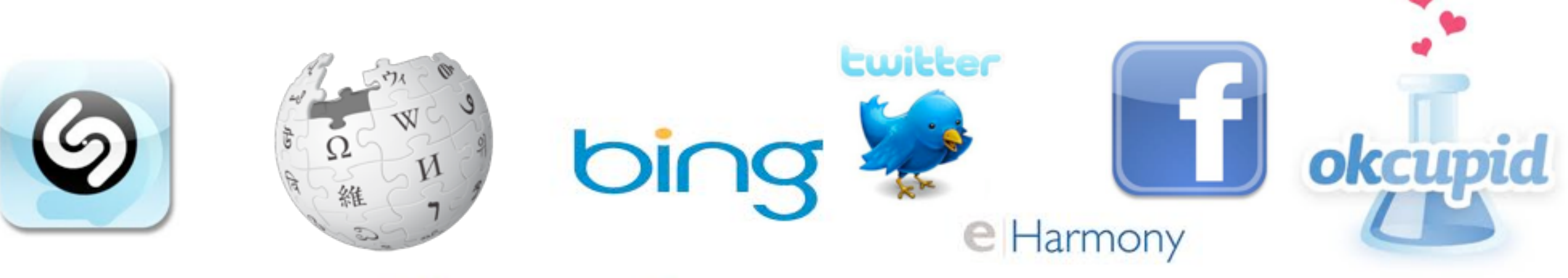

PANDORA GoOgle
mapquest $\mathrm{m}^{\mathrm{a}}$

YAFOO! ANSWERS

(iin) Linked in

yelpos

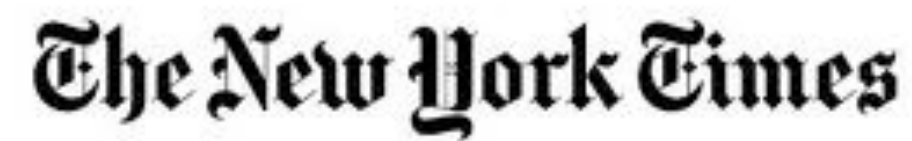

\section{flickr" (1) Picasa.}

Westlaw.

CexisNexis'

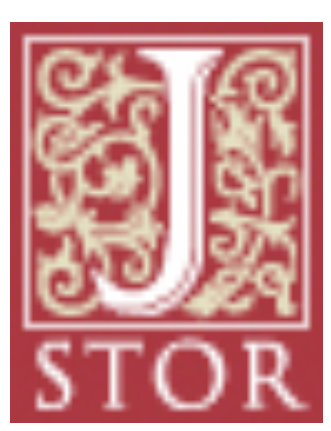

YouTube

Broadcast Yourself ${ }^{\mathrm{m}}$ 